Published in final edited form as:

Nat Methods. 2019 October 01; 16(10): 1045-1053. doi:10.1038/s41592-019-0574-9.

\title{
Nuclear pores as versatile reference standards for quantitative superresolution microscopy
}

\author{
Jervis Vermal Thevathasan ${ }^{\# 1,2}$, Maurice Kahnwald ${ }^{\# 1}$, Konstanty Cieśliński ${ }^{1}$, Philipp \\ Hoess $^{1,2}$, Sudheer Kumar Peneti ${ }^{1,3}$, Manuel Reitberger ${ }^{1,4}$, Daniel Heid ${ }^{1,5}$, Krishna \\ Chaitanya Kasuba ${ }^{1,6}$, Sarah Janice Hoerner ${ }^{1,7}$, Yiming $\mathbf{L i}^{1}$, Yu-Le $\mathrm{Wu}^{1,2}$, Markus Mund ${ }^{1,8}$, \\ Ulf Matti ${ }^{1}$, Pedro Matos Pereira ${ }^{9}$, Ricardo Henriques ${ }^{9}$, Bianca Nijmeijer ${ }^{1}$, Moritz Kueblbeck ${ }^{1}$, \\ Vilma Jimenez Sabinina ${ }^{1}$, Jan Ellenberg ${ }^{1}$, Jonas Ries ${ }^{1,{ }^{*}}$ \\ ${ }^{1}$ EMBL, Cell Biology and Biophysics, Meyerhofstr. 1, 69117 Heidelberg, Germany \\ ${ }^{2}$ Collaboration for joint PhD degree between EMBL and Heidelberg University, Faculty of \\ Biosciences \\ ${ }^{9}$ MRC-Laboratory for Molecular Cell Biology, University College London, Gower St, London, \\ WC1E 6BT, United Kingdom \\ \# These authors contributed equally to this work.
}

\section{Abstract}

Quantitative fluorescence and superresolution microscopy are often limited by insufficient data quality or artifacts. In this context, it is essential to have biologically relevant control samples to benchmark and optimize the quality of microscopes, labels and imaging conditions.

Here we exploit the stereotypic arrangement of proteins in the nuclear pore complex as in situ reference structures to characterize the performance of a variety of microscopy modalities. We created four genome edited cell lines in which we endogenously labeled the nucleoporin Nup96 with mEGFP, SNAP-tag, HaloTag or the photoconvertible fluorescent protein mMaple. We

Users may view, print, copy, and download text and data-mine the content in such documents, for the purposes of academic research, subject always to the full Conditions of use:http://www.nature.com/authors/editorial_policies/license.html\#terms

„jonas.ries@embl.de.

${ }^{3}$ Current affiliation: Centre for Bacterial Cell Biology, Institute for Cell and Molecular Biosciences, Newcastle University, Newcastle upon Tyne NE2 4AX, UK

${ }^{4}$ Current affiliation: Division of Stem Cells and Cancer, German Cancer Research Center (DKFZ), Im Neuenheimer Feld 280, 69120

Heidelberg, Germany

${ }^{5}$ Current affiliation: Department for Applied Tumor Biology, Heidelberg University Hospital, Im Neuenheimer Feld 672, 69120

Heidelberg, Germany

${ }^{6}$ Current affiliation: Eidgenössische Technische Hochschule (ETH) Zurich, Department of Biosystems Science and Engineering, Mattenstrasse 26, 4058 Basel, Switzerland

${ }^{7}$ Current affiliation: Institute of Molecular and Cell Biology, Mannheim University of Applied Sciences and Interdisciplinary Center for Neuroscience, Heidelberg University, Grabengasse 1, 69117 Heidelberg, Germany

${ }^{8}$ Current affiliation: Department of Biochemistry, University of Geneva, Science 2, Quai Ernest-Ansermet 30, 1205 Genève, Switzerland

Author contributions

J.R. conceived the approach, B.N., M.K., V.J.S., J.E., J.V.T. and U.M. generated the cell lines, J.V.T., M.K., K.C., P.H., S.K.P., M.R., D.H., K.C.K., S.J.H., Y.L., Y.W., M.M., U.M. and J.R. developed the methods, wrote the software, acquired and analyzed the data. R.H. and P.M.P. acquired the expansion microscopy data, J.V.T., M.K., P.H., M.M. and J.R. wrote the manuscript with input from all authors.

Competing financial interests

The authors declare no competing financial interests. 
demonstrate their use a) as 3D resolution standards for calibration and quality control, b) to quantify absolute labeling efficiencies and c) as precise reference standards for molecular counting.

These cell lines will enable the broad community to assess the quality of their microscopes and labels, and to perform quantitative, absolute measurements.

\section{Introduction}

Superresolution microscopy, specifically single-molecule localization microscopy (SMLM, also known as PALM ${ }^{1}$ or STORM ${ }^{2}$ ), reaches nanometer-scale optical resolution and provides structural insights into cell biological questions ${ }^{3-5}$. In SMLM, many factors have to be optimized: microscope optics, settings and stability; imaging conditions; fluorophores and labeling technologies; sample preparation; and analysis software. To date a common practice to generally optimize these factors, and to ensure comparable quality between different labs, is missing. Current algorithms for image quality control are limited as they require prior knowledge about the imaged structure ${ }^{6,7}$. Suboptimal performance in SMLM is therefore not readily detected, which severely limits biological discovery.

This is overcome by suitable reference samples, which allow a quantitative optimization of a superresolution microscopy workflow. Simulated images served as reference standards to benchmark various superresolution software ${ }^{8}$. Artificial reference structures like DNA origami ${ }^{9,10}$ allow positioning fluorophores at precise three-dimensional positions, but are limited in the choice of labels and are intrinsically different from intracellular biological structures. Cellular reference structures have included histones ${ }^{1}$, mitochondria ${ }^{11}$, and microtubules ${ }^{12}$. For instance, image resolution is often approximated by evaluating crosssectional profiles of microtubules, which requires fixation methods incompatible with other cellular structures (particularly membrane proteins) and is prone to cherry-picking. Furthermore, because these structures have highly abundant epitopes, acceptable images are obtained even for labeling efficiencies below $1 \%$. Therefore, these references are not ideal to optimize labeling efficiencies, a major factor determining image quality in SMLM.

An ideal superresolution reference structure has its fluorescent labels arranged at distances resolvable by the technique of choice and in defined numbers to allow quantifying labeling efficiencies; it uses common dyes and labeling approaches to resemble intracellular measurements; and it is present in many copies in the cell for statistical accuracy.

The nuclear pore complex (NPC) fulfills all these requirements and thus represents a quantitative reference structure. It comprises $\sim 30$ different proteins and selectively transfers macromolecules across the nuclear membrane. For the human NPC, a high resolution structural map of most nucleoporins was obtained by electron microscopy ${ }^{13}$. The NPC has been used to validate quantitative microscopy ${ }^{14-17}$ and SMLM has provided insights into its structure $^{5,18}$, highlighting its versatility.

Here, we generated cell lines where the nucleoporin Nup96 is endogenously tagged with commonly used labels. We demonstrate that imaging these cell lines yields excellent 
reference data for all experimental parameters in quantitative superresolution microscopy. We show their use to a) quantify microscope performance, resolution and calibration, b) measure absolute labeling efficiencies, c) optimize imaging conditions, and d) count protein numbers within a complex.

\section{Results}

\section{Generation of Nup96 cell lines}

We identified Nup96 as a suitable reference protein (Fig. 1a-e): It is present in 32 copies per NPC where it forms a cytoplasmic and a nucleoplasmic ring, each consisting of 16 Nup96 copies. Each ring has 8 corners that contain two Nup96, $12 \mathrm{~nm}$ apart ${ }^{13}$. The two rings are almost in register, thus the eightfold symmetry of the NPC is clearly visible (Fig. 1e).

We generated homozygous knock-in U2OS cell lines (Supplementary Figure 1, distributed by CLS Cell Line Service), where Nup96 is endogenously labeled with one of four commonly used labels: mEGFP (subsequently referred to as GFP), the photoconvertible fluorescent protein mMaple ${ }^{19}$, and the enzymatic labels SNAP-tag ${ }^{20}$ and HaloTag ${ }^{21}$. In U2OS cells, the lower nuclear envelope is flat and close to the coverslip (Fig. 1a), thus hundreds of nuclear pores are in focus in a single widefield, confocal or total internal reflection (TIRF, Supplementary Figure 2) image.

\section{Resolution and quality control}

The Nup96 corners are $10 \mathrm{~nm}$ to $100 \mathrm{~nm}$ apart, and are therefore suitable resolution standards for many microscopes. We first imaged our cells with 9 microscopy approaches (Fig. 1f-n) and obtained images with excellent signal to noise ratio for all imaging modalities. Throughout all experiments, we focused on the flat underside of the nucleus to avoid imaging tilted NPCs. For diffraction-limited techniques, NPCs act as sub-diffraction structures of defined brightness. Individual NPCs are resolved in widefield microscopy (Fig. 1f), and, with improved contrast in confocal microscopy (Fig. 1g). Airy-scan microscopy (Fig. 1h) leads to a visible, but moderate improvement in resolution (Supplementary Figure 3a). Stimulated emission depletion ( $\mathrm{STED}^{22}$ ) microscopy resolves the ring-like arrangement (Fig. 1i).

These rings are also apparent in expansion microscopy (ExM $\left.{ }^{23}\right)$ with widefield (Fig. 1j), structured illumination (Fig. 1k) and $\mathrm{SRRF}^{24}$ (Fig. 11) readout. However, they appear less complete than in STED, PALM or STORM, indicating loss of labeling during expansion. Our reference cell lines can be used to directly infer the local expansion factor from the size of the rings ${ }^{25}$ (Supplementary Figure 3c, Methods). The measured local expansion factor of 3.2 was quite different from the global expansion factor of 4.5 , indicating inhomogeneous expansion $^{26}$, that potentially complicates ExM of protein complexes.

SMLM using mMaple (PALM ${ }^{1}$ approach) shows clear rings and starts resolving the eight corners (Fig. 1m), even when imaged in living cells (Supplementary Figure 4). The highest resolution is reached using organic dyes (Fig. 1n,o), where the eight corners are very well resolved (Fig. 1p-r). The increasing lateral resolution was confirmed in the Fourier spectrum $^{27}$ (Supplementary Figure 3a) and by Fourier Ring Correlation ${ }^{28}$ (Supplementary 
Figure 3b). Superresolution approaches with even higher resolution, such as DNA-PAINT, can resolve the four individual proteins in each corner $^{29}$.

Our cell lines are also ideal to quantify the axial resolution of 3D superresolution imaging. A sufficient z-resolution allows resolving the two rings of the NPC in an axial profile, where the standard deviation of each peak is an upper limit for the experimental localization precision (Fig. 1s, Fig. 2h,j).

\section{Microscope calibration}

The calibration of a superresolution microscope can be verified by comparing measured distances between Nup96 clusters to the ground truth, which we measured using an automated SMLM microscope with a precisely calibrated pixel size (Methods). The average radius of the NPC was $\mathrm{R}=53.7 \pm 2.1 \mathrm{~nm}$ in Nup96-SNAP cells (Fig. 2a-c, all values are mean \pm standard deviation (SD) unless otherwise noted), and similar in the other cell lines (Supplementary Figure 5), except for antibody-labeled Nup96-GFP with $\mathrm{R}=64.3 \pm 2.6 \mathrm{~nm}$. The cytoplasmic and nucleoplasmic rings were $49.3 \pm 5.2 \mathrm{~nm}$ apart (Fig. 2d-f, Methods) and azimuthally shifted by $8.8^{\circ} \pm 0.6^{\circ}$ (Fig. $2 \mathrm{~g}, \mathrm{~h}$, Methods).

In addition to the pixel size, our cell lines can be used to verify the axial calibration in 3D SMLM. This is challenging, as aberrations or imperfect PSF models can lead to depthdependent localization errors, especially when using oil objectives ${ }^{30}$. Moreover, the refractive index difference between oil and the aqueous sample leads to an image compressed in $\mathrm{z}$. This can be corrected by applying a refractive index mismatch factor $(\mathrm{RIMF})^{12}$, which however is usually not precisely known and difficult to calibrate.

Here, we used our tagged Nup96 cell lines to validate the z-calibration of our astigmatic SMLM microscope by measuring the distance between the two rings in thousands of NPCs in 3D. The average distance was $d=42.1 \pm 1.1 \mathrm{~nm}$ (Fig. 2i, based on a RIMF of 0.8), and thus smaller than the true value of $49.3 \mathrm{~nm}$. Furthermore, the distance between the rings was correlated to the distance between NPC and coverslip (Supplementary Figure 6a), indicating aberrations. After correcting for these aberration-induced fitting errors with a method we recently developed ${ }^{30}$, the z-dependence was reduced (Supplementary Figure 6b) and the average corrected distance is $d=49.8 \pm 1.9 \mathrm{~nm}$. Based on these results we could calibrate the RIMF to be 0.79 .

\section{Effective labeling efficiencies}

Besides the localization precision, the information content of SMLM images critically depends on how densely the imaged structures are decorated with fluorophores. This can be described by the effective labeling efficiency (ELE), which represents the fraction of target proteins that carry a fluorophore that is detected as a useable localization (i.e. brightness above background, fitted with acceptable confidence). By definition, this parameter takes into account conjugation efficiency between dye and ligand, bleaching during initial offswitching and the fraction of non-functioning fluorophores.

Previously, the maturation efficiency of photoactivatable proteins has been estimated using receptors on the cell surface ${ }^{14}$ or by mathematical modeling of fluorophore photophysics ${ }^{31}$, 
and binding efficiencies of anti-GFP antibodies were measured ${ }^{15}$. Altogether however, a robust approach to measure the absolute ELE of common labeling strategies inside cells is still missing, limiting a systematic optimization of image quality in SMLM.

Our Nup96 cell lines provide a simple assay to directly measure absolute ELEs. When the ELE is low, NPCs appear as incomplete rings with missing corners. Thus, by statistically analyzing the number of corners of many NPCs, we can infer the absolute ELE. Here, we developed a workflow to automatically determine the number of corners in hundreds of NPCs (Fig. 3, Methods). The variability of the measured ELE between cells and biological replicates was typically smaller than $10 \%$ (SD, Fig 3i). Using simulations (Supplementary Figure 7), we showed that this approach is robust over a large range of ELEs, localization precisions and number of re-activations. Only for very high $(>90 \%)$ and very low $(<10 \%)$ ELE the precision is reduced and quantification of low affinity binders might necessitate costaining with e.g. WGA (wheat germ agglutinin) for unbiased segmentation.

Using this workflow, we systematically compared the ELEs of different anti-GFP nanobodies ${ }^{32}$, and SNAP-tag and HaloTag ligands with different organic dyes (Fig. 3i, Supplementary Table 1). Here, we observed the highest ELEs of $\sim 74 \%$ using a commercial mixture of two different anti-GFP nanobodies. Other monoclonal commercial anti-GFP nanobodies achieved $\sim 62 \%$, while anti-GFP nanobodies that we generated in the lab showed a lower ELE of $45 \%$, which was further reduced to $25 \%$ after 2 years of storage in the fridge. Indirect immunofluorescence reached an ELE of $65 \%$.

For Nup96-SNAP labeled with BG-AF647 we achieved an ELE of 58\%. Stored SNAP-tagstained samples were stable over years (shown for Nup107-SNAP in Supplementary Figure 8) with only minor loss in ELE, facilitating prolonged regular usage of these standard samples.

Using HaloTag, we achieved lower ELEs of $21 \%$ - $40 \%$ with four different ligands. While the photo-activatable ligand PA-JF549 ${ }^{33}$ showed no specific labeling in fixed cells, it could be used for live-cell labeling with an ELE of $21 \%$. Interestingly we note that, while a single AF647 dye is localized on average $3.4 \pm 0.4$ times, PA-JF549 produces on average $1.3 \pm 0.1$ localizations and thus shows little blinking. This is well-suited to investigate protein clustering as it reduces false positives caused by re-activation of fluorophores.

Simulations (Supplementary Figure 7) indicated that our approach of quantifying the ELE works even when the individual corners are not always resolved. Thus, we extended our analysis to the photoconvertible fluorescent protein mMaple. We found an ELE of 58\%, indicating that even though $100 \%$ of all Nup96 are fused to mMaple, about $40 \%$ of them are not detected as a localization. This is likely due to improper folding, insufficient brightness or incomplete photoconversion, in line with previous reports ${ }^{14,31}$.

Taken together, this assay provides an easy way for any lab using SMLM to monitor the ELEs of their labeling reagents, thus avoiding the use of sub-optimal labels. 


\section{Imaging conditions}

Numerous factors influence image quality in SMLM, including imaging buffers, laser intensities, exposure times, filters, and settings in the analysis software. To find optimal conditions, these factors are varied while optimizing various read-outs for quality, including fluorophore brightness, low background, on-times, duty cycle, localization precision, ELE, number of re-activations, imaging speed or stability of imaging buffers. Such optimization requires a robust standard sample with small variability to allow detection of subtle changes.

We used our reference standards to read out these parameters, in order to compare various common imaging conditions (Table 1, Supplementary Figure 9). We confirmed that AF647, compared to the MEA buffer, shows increased brightness and number of localizations per fluorophore in $\mathrm{BME}^{34}$, reduced brightness and number of localizations per fluorophore in sulfite buffer ${ }^{35}$, and no substantial change in $\mathrm{D}_{2} \mathrm{O}^{36}$. Interestingly, we found that a high ELE correlates with a large number of localizations per fluorophore, possibly due to less bleaching during the first switching-off cycle. Finally, we found that PFA fixation did not change mMaple photophysics or ELE and confirmed that mMaple becomes brighter in $\mathrm{D}_{2} \mathrm{O}$ compared to $\mathrm{H}_{2} \mathrm{O}^{37}$.

\section{Counting of proteins}

Knowing the stoichiometry of a multi-protein assembly is essential for functional studies. Converting gray values from a fluorescence microscopy image to absolute protein numbers requires careful calibration of the microscope. The Nup96-GFP cell line is well suited for this task, as the majority of nuclear pores are resolved even in diffraction-limited microscopy (Fig. 1f,g, Fig. 4a). Thus, we can calibrate precisely how bright 32 GFP-labeled proteins are, and use this calibration to determine the unknown abundance of a different GFP-labeled protein. For validation, we chose Nup107, another nucleoporin present in 32 copies per $\mathrm{NPC}^{38}$. In a simple brightness analysis we evaluated the intensity of the brightest pixel of a local intensity maximum as a measure for the brightness of the NPC and found similar average values for Nup96-GFP and Nup107-GFP 39 (Fig. 4a-d).

SMLM allows counting of proteins in dense structures, but relating the number of localizations to the number of proteins is not trivial. Incomplete labeling leads to undercounting, while repeated fluorophore re-activation induces overcounting. Previous approaches attempted to calibrate blinking and other photophysical properties of fluorophores ${ }^{40-42}$, which however cannot account for long-lived dark states and incomplete labeling or maturation. Furthermore, a variety of counting references have been developed including self-assembling oligomers ${ }^{16}$, DNA-structures ${ }^{15}$, receptors ${ }^{14}$, or a combination of fluorescent protein oligomers ${ }^{31,43,44}$. While this is a powerful approach, a major limitation is the need for faithful segmentation, which is often strongly dependent on algorithmic parameters. Background localizations or incompletely assembled or labeled reference structures lead to an underestimation of the reference brightness, whereas fusion of doublestructures or a cutoff during segmentation and thus loss of small structures leads to an overestimation. Moreover, the detection probability of a fluorophore depends on its zposition, which renders cytoplasmic reference structures less accurate. 
Our cell lines overcome many of these limitations. NPCs have a characteristic shape and large size, never overlap and are thus easy to segment. They are abundant for improved statistics and are at defined z-positions. To validate the use of Nup96-mMaple as a counting reference standard, we generated a stable HEK293T cell line where Nup107-mMaple was overexpressed, while endogenous Nup107 was knocked down (Supplementary Figure 10). We found $32.1 \pm 2.5$ Nup107 molecules per NPC (Fig. 4e-h), highlighting the consistency of this counting approach.

Accurate counting requires complete tagging of all target proteins, which is laborious in mammalian cells. We thus extended our counting references to $S$. cerevisiae, where homologous recombination allows for fast and efficient endogenous labeling (Supplementary Table 2). We chose the nucleoporin Nup188 as the reference standard, which is present in 16 copies per NPC ${ }^{45,46}$. We endogenously tagged Nup188 with mMaple in yeast cells that additionally express a GFP-marker for identification. This allowed us to simultaneously image reference and target cells in the same field of view (Fig. 4i,j). We first validated this approach by counting Nup82, which is present also in 16 copies $^{45,46}$, and by counting Nup82 and Nup188 molecules together within a strain where both were tagged with mMaple (Fig. 4k,l). The measured copy numbers of $15.7 \pm 0.7$ and $30.3 \pm 1.7$ agree well with their expected values of 16 and 32, respectively.

We then counted the nucleoporins Nup192 and Nic96 (Fig. 4m). Nup192 was found in 16.4 \pm 1.8 copies per NPC, agreeing with previous reports ${ }^{45,46}$. Intriguingly, for Nic 96 we found $26.8 \pm 1.2$ copies when Nic96 was tagged at the $\mathrm{C}$-terminus, contradicting previous reports that found 32 copies of $\mathrm{Nic} 96^{45}$. It was recently proposed that $\mathrm{C}$-terminal tagging impedes Nic96 function ${ }^{46}$, and indeed we measured $33.0 \pm 2.0$ copies of N-terminally tagged Nic96. When we introduced an additional GFP tag at the C-terminus of Nup49, which interacts with the $\mathrm{C}$-terminus of Nic96, we again measured only $27.8 \pm 1.7$ copies even for $\mathrm{N}$-terminally tagged Nic96. Our findings demonstrate the reproducibility of our method, and emphasize the risk of tagging artifacts. Careful quantification of proteins with our counting approach offers an experimental avenue to systematically control for them.

As yeast cells duplicate every $\sim 2 \mathrm{~h}$, maturation times of fluorescent proteins must be considered. Assuming a maturation time of 48 minutes for mMaple ${ }^{47}$ results in $28 \%$ of unmatured mMaple in the steady-state (Methods). To experimentally test the influence of maturation on our measurements, we stopped protein synthesis by cycloheximide (CHX), reasoning that mMaple synthesized before the treatment should mature to completion. The measured increase in the number of localizations by $11 \pm 6 \%$ was less than estimated above, hinting either to a delay in incorporation of Nup188 into the NPC, degradation of newly synthesized Nup188, to a faster maturation time than previously estimated, or to a maturation of mMaple after fixation. Generally, we recommend using this or a related approach to stop protein synthesis whenever the lifetime of the target protein is short or unknown. 


\section{Discussion}

By homozygously labeling Nup96 with four common tags, we generated reference standards for a variety of important applications in microscopy. Shared together with the software to perform all analyses, the cell lines enable the community to benchmark resolution and calibration of their microscopes, to optimize imaging conditions with high sensitivity, to determine effective labeling efficiencies of their labels and to count protein copy numbers.

The assays presented here are robust and reproducible due to the stereotypic architecture of the NPC. Interestingly, we observed some biological variation in the dimension of the NPC structure (Fig. 2), in line with previous reports by electron microscopy ${ }^{48}$. Thus, a statistical analysis of many NPCs is needed for accurate parameter estimates. This heterogeneity might be interesting with respect to nuclear pore biology, and the data accompanying this manuscript could be the basis for such analysis. Although present, this structural variability is still smaller than that of 3D DNA origami standard samples ${ }^{10,49}$. For some labeling protocols, we observed a cell-to-cell variability of the ELE with a subset of cells showing reduced labeling, stressing the need for replicates and optimal sample preparation. Finally, artifacts (e.g. by drift or over-activation) or insufficient localization precision impede accurate determination of ELEs.

Intracellular labeling with SNAP-tag or HaloTag did not result in complete labeling as is possible in vitro ${ }^{20,21}$. We observed that the choice of linker and dye strongly affected the ELE, in line with a recent report ${ }^{50}$. Also, with anti-GFP nanobodies labeling was not complete. Incomplete labeling could arise from incomplete folding of the enzymatic tags, inhibition of the tags by fixatives or intracellular components, or by incomplete activation and detection of the fluorophores, imperfect ligands, or bleaching during the initial offswitching step in SMLM, which warrants further investigation and optimization.

It should be stressed that the ELE measured on NPCs is not necessarily equal to ELEs on other target proteins owing to differences in epitope accessibility and local environment. However, we expect that imaging protocols optimized on Nup96 cell lines will be suitable for other structures, and that labels that achieve only a low ELE on Nup96 will also perform poorly on other targets.

As counting reference standards, NPCs are advantageous to small and globular structures due to the ease of segmentation and their defined z-positions. However, some fundamental limitations still apply to any reference-based approach: both target and reference structures need to be in focus and well segmented to exclude overlapping structures and background localizations from outside the imaging plane, and all target proteins need to carry a label, thus requiring endogenous protein tagging. Furthermore, incomplete maturation of photoconvertible proteins has been reported before ${ }^{14}$. Thus, protein turnover and maturation rates in the cell need to be accounted for, and protein synthesis might need to be stopped for a limited time to ensure all labels are matured. Finally, counting with fluorescent proteins, as we have used here, is preferable to counting with external labels, where different epitope accessibilities of the tags between reference and target need to be considered. 
Our Nup96 cell lines optimally complement the current standard sample for SMLM, i.e. immunolabeled microtubules, as they have a defined stoichiometry and 3D arrangement of the fluorophores and are compatible with most common labeling approaches. Together with the community we will extend the collection of Nup96 cell lines to other fluorescent proteins and peptide tags. We expect that they will find widespread use in many labs for optimization, quality control and counting and that they become the gold standard to quantify effective labeling efficiencies of new dyes and labels.

\section{Methods}

\section{Generation of CRISPR cell lines}

All cell lines are distributed by Cell Line Services (CLS, clsgmbh.de, Nup96-SNAP \#300444, Nup96-Halo \#300448, Nup96-mEGFP \#300174, Nup96-mMaple \#300461).

Genome editing was performed using CRISPR-Cas9D10A nickase as described in Koch et al. ${ }^{51}$ The gRNA sequences for Nup96 C-term are as follows, sense: 5'GTTGGGAGCCTGTGAGCCCC-3' and antisense: 5'-CAGTTCTCGCAGATAGGACT-3'.

The synthetic gene pNup96-mEGFP donor plasmid encoding for left $(1.1 \mathrm{~kb})$ and right $(0.8$ $\mathrm{kb}$ ) homology arms for the C-terminus of Nup96 was assembled from synthetic oligonucleotides and/or PCR products. A linker sequence (5' ACTAGTCGACGGTACCGCGGGCCCGGGATCCACCGGCCGGTCGCCACC 3') between the left homology arm containing multiple cloning sites was inserted to aid the generation of donor plasmids encoding for other tags. The fragment was inserted into the pMA-RQ (ampR) vector backbone.

Donor plasmids encoding for mMaple ${ }^{19}, \mathrm{SNAP}_{\mathrm{f}} \operatorname{tag}^{52}$ (NEB) and HaloTag (Promega) were generated by swapping out mEGFP using restriction enzymes EagI-HF and NheI-HF(NEB). Tag-sequences can be found in the supplementary information.

Southern blotting of Nup96-Southern blotting was performed in accordance Koch et al. ${ }^{51}$ Genomic DNA was prepared using the Wizard Genomic DNA Purification kit (Promega) and digested with $S s p I-H F$ and $M f e I-H F(\mathrm{NEB})$. The probe sequences used are as follows:

Nup96 C-term:

$\left(5^{\prime}\right.$

TCCAGTTTCTCTCTGCCACATCCACCTGTTTAAATTATCTACATGGCTTGTGATTTT TCAGGATTTATTACTGTTTTGTGTTTTCTTATTTATTTTCTATCAGTTTCATGAGAGC AAATAACCTGTCTTGCTCTTGATCCTCCTGCCCCCTGCACACAGCTTTTTTGGTGT TTTAGAAAAGGCTATAAACTTGGAGTCAGGGGACCT-3');

mEGFP:

$\left(5^{\prime}\right.$

CACATGAAGCAGCACGACTTCTTCAAGTCCGCCATGCCCGAAGGCTACGTCCAGG 
AGCGCACCATCTTCTTCAAGGACGACGGCAACTACAAGACCCGCGCCGAGGTGA AGTTCGAGGGCGACACCCTGGTGAACCGCATCGAGCTGAAGGGCATCGACTTCA AGGAGGACGGCAACATCCTGGGGCACAAGCTGGAGTACAACTACAACAGCCACA ACGTCTATATCATGGCCGACAAGCAGAAGAACGGCATCAAGGTGAACTTCAAGAT CCGCCACAACATCGAGGACGGCAGCGTGCAGCTCGCCGACCACTACCAGCAGAA CACCC-3');

mMaple:

$\left(5^{\prime}\right.$

AGCATGACCTACGAGGACGGCGGCATCTGCATCGCCACCAACGACATCACAATGG AGGAGGACAGCTTCATCAACAAGATCCACTTCAAGGGCACGAACTT-3');

SNAPtag:

$\left(5^{\prime}\right.$

AAAGACTGCGAAATGAAGCGCACCACCCTGGATAGCCCTCTGGGCAAGCTGGAA CTGTCTGGGTGCGAACAGGGCCTGCACCGTATCATCTTCCTGGGCAAAGGAACAT CT-3');

HaloTag:

$\left(5^{\prime}\right.$

TGCATTGCTCCAGACCTGATCGGTATGGGCAAATCCGACAAACCAGACCTGGGTT ATTTCTTCGACGACCACGTCCGCTTCATGGATGCCTTCATCGAAGC-3')

siRNA silencing of Nup96 in U2OS-To test specificity of the anti-Nup98 antibody, U2OS cells were seeded onto a $35 \mathrm{~mm}$ cell culture dish. $48 \mathrm{~h}$ after seeding, MISSION® esiRNA Human nup98 (esirna1) (Sigma, EHU087381-20ug, Lot: BEV) was introduced using lipofectamine 2000 (life technologies). $48 \mathrm{~h}$ after transfection the cell layer was scrapped and cell lysate was collected for western blot analysis.

Western blotting of Nup96-U2OS cell lysates were collected in Pierce RIPA buffer (Cat\#89900; Lot no. NF170965; ThermoFisher Scientific) supplemented with Complete protease inhibitors (Roche) and phenylmethanesulfonylfluoride (PMSF). Cell lysate protein concentration was determined using Pierce BCA protein assay kit (Cat\#23225; Lot no.

QI223168; ThermoFisher Scientific). $50 \mu \mathrm{g}$ of cell lysate was loaded onto a 4-12\% gradient gel and ran at $165 \mathrm{~V}$ constant for 45-60 min in 1X MOPS-SDS buffer (NuPAGE) at room temperature (RT). Proteins were then transferred to a PVDF membrane at $15 \mathrm{~V}$ constant for 60 min in cold 1X transfer buffer supplemented with $10 \%(\mathrm{v} / \mathrm{v})$ methanol (Bolt ${ }^{\mathrm{TM}}$ ) at RT. Membranes were then blocked in 10\% (w/v) milk in TBS-T pH 7.6 for 1 hour at RT. After blocking, membranes were incubated in 1:2000 diluted primary antibody (pAb anti-Nup98, Cat\#NB1000-93325; LotA1; Novus) in 3\% (w/v) BSA in TBS-T at $4{ }^{\circ} \mathrm{C}$ overnight.

Membranes were then incubated in 1:10000 diluted secondary antibody in 5\% (w/v) milk in TBS-T for 1 hour at RT. Chemiluminescence reagents were added to the membrane with subsequent film exposure. 


\section{Sample preparation}

\section{Buffers}

Sample seeding-Prior to seeding of cells, high-precision $24 \mathrm{~mm}$ round glass coverslips (No. 1.5H; Cat\#117640; Marienfeld, Lauda-Königshofen, Germany) were cleaned by placing them overnight in a methanol/hydrochlorid acid (50/50) mixture while stirring. Following that, the coverslips were repeatedly rinsed with water until they reached a neutral $\mathrm{pH}$. They were then placed overnight into a laminar flow cell culture hood to dry them before finalizing the cleaning of coverslips by UV-irradiation for $30 \mathrm{~min}$.

For superresolution microscopy, homozygous endogenously tagged cells were seeded on clean glass coverslips two days prior fixation in such a way, that they reach a confluency of about $50-70 \%$ on the day of fixation. For diffraction limited techniques, cells were seeded on $35 \mathrm{~mm}$ cell culture dishes with a $10 \mathrm{~mm}$ glass bottom insert (Cat\#627860; Greiner Bio-One) instead. Cells grew on the coverslip or the $35 \mathrm{~mm}$ cell culture dish in growth medium (DMEM [Gibco; \#11880-02] containing 1x MEM NEAA [Cat\#11140-035; Gibco], 1x GlutaMAX [Cat\#35050-038; Gibco] and 10\% [v/v] fetal bovine serum [Cat\#10270-106; Gibco]) for approximately two days at $37{ }^{\circ} \mathrm{C}$ and $5 \% \mathrm{CO}_{2}$. Before further processing, the growth medium was aspirated, and samples were rinsed two times with PBS to remove dead cells and debris. Unless otherwise stated, all experiment replicates were performed on cells of different passage seeded on coverslips. Within each coverslip multiple cells were imaged.

Expansion microscopy (proExM)-Expansion of samples was performed as described elsewhere ${ }^{55}$. Briefly, monomer solution (1x PBS, $2 \mathrm{M} \mathrm{NaCl}, 8.625 \%$ [w/w, Sigma] sodium acrylate, $2.5 \%$ [w/w, Sigma] acrylamide, $0.15 \%$ [w/w, Sigma] $N, N^{\prime}$ -

methylenebisacrylamide) was mixed and cooled to $4{ }^{\circ} \mathrm{C}$ before use. Ammonium persulfate (APS, BIORAD) initiator and tetramethylethylenediamine (TEMED, Sigma) accelerator were added to the monomer solution up to $0.2 \%$ (w/w) each. Samples on coverslips were incubated with the monomer solution plus APS/TEMED in a humidified $37{ }^{\circ} \mathrm{C}$ incubator for $1 \mathrm{~h}$ for gelation. Proteinase $\mathrm{K}$ (New England Biolabs) was diluted 1:100 to 8 units $/ \mathrm{mL}$ in digestion buffer (50 mM Tris/HCl pH 8, 1 mM EDTA, 0.5\% [v/v] Triton X-100, $1 \mathrm{M} \mathrm{NaCl}$, Sigma) and incubated with the gels fully immersed in proteinase solution overnight at $23{ }^{\circ} \mathrm{C}$. Digested gels were next placed in excess volumes of double deionized water for 3-4 $\mathrm{h}$ to expand (water changed every $30 \mathrm{~min}$ ), until the size of the expanding sample plateaued. A small piece of the expanded sample was mounted in an ATTOFLUOR chamber (ThermoFisher Scientific) on $18 \mathrm{~mm}$ PLL (Sigma) coated coverslips (Marienfeld) and covered with low-melting agarose (Sigma). To determine the level of sample expansion, the average size of nuclei pre- and post-expansion was measured.

\section{Nanobody labeling of Nup96-mEGFP fusion proteins-U2OS-Nup96-mEGFP}

cells, either prepared on glass coverslips for superresolution measurements or $35 \mathrm{~mm}$ cell culture dishes for diffraction limited techniques, were stained according to a protocol previously described by Pleiner and colleagues ${ }^{53}$. For this, samples were prefixed for $30 \mathrm{~s}$ in TRB containing 2.4\% (w/v) formaldehyde (FA), followed by washing twice in TRB for 5 min each. Plasma membrane-specific permeabilization was achieved by 8 min incubation on ice in TRB containing $25 \mu \mathrm{g} / \mathrm{mL}$ digitonin (Cat\#D141; Sigma Aldrich). Samples were 
washed twice for $5 \mathrm{~min}$ in TBA. First round of staining was achieved by incubating the samples upside-down in a drop of TBA containing $100 \mathrm{nM}$ of anti-GFP nanobodies (NanoTag Biotechnologies, FluoTag-Q [Cat\#N0301] or FluoTag-X4 [Cat\#N0304], either conjugated to AF647, CF680 or STAR 635P) for $30 \mathrm{~min}$ on ice. Residual nanobodies were rinsed away in TBA twice for 5 min each before cells were further fixed in TBA containing 3\% (w/v) FA for 10 min followed by two additional washing steps in TBA for 5 min each. Permeabilization of the nuclear envelope was facilitated by 3 min incubation in PB. Samples were washed twice in PBS for 5 min each before exposing them again upside-down onto a drop of anti-GFP nanobodies (50 nM in TBA, same nanobodies as in the first round of staining) for $30 \mathrm{~min}$ on ice. Finally, weakly bound and unbound nanobodies were rinsed off in PBS twice for $15 \mathrm{~min}$. For STED-imaging, FluoTag-X4-STAR 635P stained samples were mounted upside-down on glass microscopy slides (ThermoFisher Scientific) using Mowiol (Calbiochem). Edges were further sealed by nail polish and then dried overnight at RT.

Indirect immunostaining of Nup96-mEGFP fusion proteins-To fix U2OS-Nup96mEGFP cells on the glass coverslips, cells were prefixed in FB for $30 \mathrm{~s}$ before incubating them 3 min in $0.1 \%$ Triton X-100 in PBS and washing twice for 5 min each in PBS. Fixation was completed in FB for $20 \mathrm{~min}$. Samples were quenched for $5 \mathrm{~min}$ in QS and then washed twice in PBS for 5 min each. Fixed cells were then further permeabilized using $0.2 \%$ Triton X-100 in PBS for $10 \mathrm{~min}$, followed by two more washing steps in PBS for 5 min each before blocking in 2\% (w/v) BSA in PBS for $1 \mathrm{~h}$. Binding of primary rabbit anti-GFP antibodies (Cat\#598; MBL International) to Nup96-GFP fusion proteins was achieved by placing the coverslips overnight upside-down onto a drop of primary antibody solution (diluted 1:250 in PBS containing $2 \%[\mathrm{w} / \mathrm{v}] \mathrm{BSA}$ ) at $4{ }^{\circ} \mathrm{C}$. Weakly and unbound primary antibodies were subsequently rinsed away with three washing steps in PBS for 5 min each. Secondary antibody labeling was achieved by placing the samples upside-down onto a drop of antirabbit antibodies with conjugated AF647 dye (custom made, diluted 1:300 in PBS containing $2 \%[\mathrm{w} / \mathrm{v}] \mathrm{BSA}$ ) for $1 \mathrm{~h}$ at RT. Residual secondary antibody was removed by washing thrice with PBS for 5 min.

HaloTag labeling of fixed cells-U2OS-Nup96-Halo cells were stained on previously prepared coverslips using a slightly modified version of the nanobody labeling protocol described above ${ }^{53}$. Instead of staining the samples in two separate rounds of nanobodies (100 $\mathrm{nM}$ in round 1 and $50 \mathrm{nM}$ in round 2), the samples were incubated in HaloTag dye buffer (5 $\mu \mathrm{M}$ of Cy5-HaloTag ligand [Lavis Lab, HHMI Janelia Research campus] or HaloTag-ligand-O2-AF647/HaloTag-ligand-O4-AF647 [custom substrates from Peps4LS, Heidelberg] in TBA) for $1 \mathrm{~h}$ at RT in both incubation steps. All other steps were performed in accordance to the above described protocol.

HaloTag live labeling-Coverslips covered in an approximately 50-70\% confluent layer of U2OS-Nup96-Halo were incubated in pre-warmed growth medium containing PA-JF549HaloTag ligand $(250-5000 \mathrm{nM}$ were tested without significant difference in labeling efficiency; Lavis Lab, HHMI Janelia Research campus) at $37{ }^{\circ} \mathrm{C}$ and $5 \% \mathrm{CO}_{2}$ for $1 \mathrm{~h}$. The samples were subsequently rinsed thrice in pre-warmed PBS and incubated in pre-warmed growth medium without dye for $1 \mathrm{~h}$ at $37{ }^{\circ} \mathrm{C}$ and $5 \% \mathrm{CO}_{2}$ to wash off non-covalently bound 
dye. Following that, the samples were rinsed three times in PBS before prefixing them at RT for $30 \mathrm{~s}$ in FB. Permeabilization was facilitated in PB for 3 min before completing the fixation process for $30 \mathrm{~min}$ in FB. Subsequently, FA was quenched by incubating the coverslip for $5 \mathrm{~min}$ in QS. Sample preparation was finalized by washing twice in PBS for 5 min each.

SNAP-tag labeling of fixed cells-U2OS-Nup96-SNAP cells were prefixed for $30 \mathrm{~s}$ in FB before permeabilization in $\mathbf{P B}$ for $3 \mathrm{~min}$. To complete fixation, samples were incubated for $30 \mathrm{~min}$ in FB. FA was subsequently quenched in QS for $5 \mathrm{~min}$ before washing the coverslip twice for $5 \mathrm{~min}$ in PBS. To reduce unspecific binding, the sample was incubated for 30 min with Image-iT FX Signal Enhancer (ThermoFisher Scientific) before staining in SNAP dye buffer (1 $\mu$ M BG-AF647 [New England Biolabs; \#S9136S], $1 \mu$ M DTT in 0.5\% $[\mathrm{w} / \mathrm{v}] \mathrm{BSA}$ in PBS) for $2 \mathrm{~h}$ at RT. To remove unbound dye, coverslips were washed three times in PBS for 5 min each.

Fixation of mMaple tagged cell lines-Glass coverslips prepared with U2OS-Nup96mMaple or HEK-Nup107-mMaple cells were prefixed for $30 \mathrm{~s}$ in FB before incubation in PB for $3 \mathrm{~min}$. To complete fixation, samples were incubated for $30 \mathrm{~min}$ in FB. FA was subsequently quenched in QS for $5 \mathrm{~min}$ before washing the coverslip twice for $5 \mathrm{~min}$ in PBS.

Strain \& sample preparation for yeast-For protein counting in Saccharomyces cerevisiae, the respective proteins (Nup188, Nup82, Nup192, Nic96, Nup49 and Abp1) were endogenously tagged on the C-terminus by homologous recombination. Shortly, we constructed plasmids encoding mMaple and different selectable markers by standard molecular biology methods ${ }^{56}$. The cassette containing a peptide linker, mMaple and the selectable marker was amplified by PCR and transformed into competent yeast cells. Yeast cells were plated on selective plates, grown for 2-3 days until single colonies were obtained. Correct tagging was confirmed by colony PCR and imaging.

$\mathrm{N}$-terminal labeling of Nic96 was performed seamlessly ${ }^{57}$. First, a cassette was amplified by PCR from a vector that contains the first $180 \mathrm{bp}$ of mMaple, the selectable marker for the expression of the URA3 gene and a promoter for the tagged gene of interest surrounded by two I-SceI restriction sites and full-length mMaple. This cassette was transformed into yeast cells that express I-SceI under control of a galactose inducible promotor. After correct integration was confirmed by colony PCR, the strain was cultivated on plates containing galactose to induce the expression of I-SceI and resistance cassette loopout. Successful excision was counterselected on plates containing 5-fluoroorotic acid.

For immobilization of yeast, the coverslips were coated with concanavalin A (ConA; Cat\#C2010; Sigma-Aldrich). For this, the coverslips were cleaned overnight in a 1:1 mixture of methanol and hydrochloric acid, washed 3 times with $\mathrm{dH}_{2} \mathrm{O}$ and plasma-cleaned. Next, 20 $\mu \mathrm{l}$ of $4 \mathrm{mg} / \mathrm{mL}$ ConA in PBS was pipetted onto the coverslip and spread, incubated under a humidified atmosphere for $30 \mathrm{~min}$ and then dried. 
For super-resolution imaging, the respective strains were grown at $30{ }^{\circ} \mathrm{C}$ shaking at $220 \mathrm{rpm}$ in synthetic complete medium without tryptophan (SC-Trp) to reduce autofluorescence. A 4 $\mathrm{mL}$ overnight culture was inoculated from a single colony on a freshly restreaked plate. In the morning of the experiment, the culture was diluted to an optical density $\left(\mathrm{OD}_{600}\right)$ of 0.25 in $10 \mathrm{~mL}$ of SC-Trp medium and cultured for approximately 3 more hours to logarithmic phase. To inhibit protein synthesis, CHX was added to a final concentration of $250 \mu \mathrm{g} / \mathrm{mL}$ (from a $50 \mathrm{mg} / \mathrm{mL}$ stock solution) for the last hour of growth. Then, cells from the reference strain (Nup188-mMaple Abp1-mEGFP) and the respective target strain were mixed in a 1:1 ratio and spinned down in a table top centrifuge ( $3 \mathrm{~min}$ at $1000 \mathrm{~g}$ and RT), resuspended in about $200 \mu \mathrm{l}$ of residual medium and pipetted onto a ConA-coated coverslip. All subsequent steps were carried out in the dark to prevent pre-conversion of mMaple. After allowing the cells to settle for $20 \mathrm{~min}$, the coverslips were fixed in fixation solution (4\% [w/v] FA, $2 \%$ [w/v] sucrose, in PBS) for $15 \mathrm{~min}$. Subsequently, remaining FA was quenched by washing twice for $5 \mathrm{~min}$ in QS. After washing 3 time with PBS for 5 min each, the coverslip was ready for imaging. The sample was mounted on a custom sample holder in imaging buffer (50 mM Tris $\mathrm{pH} 8$ in $95 \%[\mathrm{v} / \mathrm{v}] \mathrm{D}_{2} \mathrm{O}$ ) and subjected to SMLM.

\section{Microscopy}

Microscope setup and imaging-All SMLM data were acquired on a custom built widefield setup described previously ${ }^{4,58}$. Briefly, the free output of a commercial laser box (LightHub; Omicron-Laserage Laserprodukte, Dudenhofen, Germany) equipped with Luxx 405, 488 and 638 and Cobolt 561 lasers and an additional $640 \mathrm{~nm}$ booster laser (iBeam smart, Toptica, Gräfelfing, Germany) were collimated and focused onto a speckle reducer (Cat\#LSR-3005-17S-VIS; Optotune, Dietikon, Switzerland) before being coupled into a multi-mode fiber (Cat\#M105L02S-A; Thorlabs, Newton, NJ, USA). The output of the fiber was magnified by an achromatic lens and focused into a sample to homogeneously illuminate an area of about $1000 \mu \mathrm{m}^{2}$. Alternatively, a single-mode fiber (Omicron, LightHUB) could be plugged into the output of the laserbox to allow TIRF imaging. The laser is guided through a laser cleanup filter (390/482/563/640 HC Quad; AHF, Tübingen, Germany) to remove fluorescence generated by the fiber. Emitted fluorescence was collected through a high-numerical-aperture (NA) oil-immersion objective (160x/1.43-NA; Leica, Wetzlar, Germany), filtered by a bandpass filter (525/50 [Cat\#FF03-525/50-25, Semrock, Rochester, NY, USA] for mEGFP; 600/60 [Cat\#NC458462, Chroma, Bellows Falls, VT, USA] for mMaple and PA-JF549 and 700/100 [Cat\#ET700/100m, Chroma] for AF647, Cy5 and CF680) and imaged onto an Evolve512D EMCCD camera (Photometrics, Tucson, AZ, USA). The $\mathrm{z}$ focus was stabilized by an IR-laser that was totally internally reflected off the coverslip onto a quadrant photodiode, which was coupled into closed-loop feedback with the piezo objective positioner (Physik Instrumente, Karlsruhe, Germany). Laser control, focus stabilization and movement of filters was performed using a field-programmable gate array (Mojo; Embedded Micro, Denver, CO, USA). The pulse length of the $405 \mathrm{~nm}$ (laser intensity $27.5 \mathrm{~W} / \mathrm{cm}^{2}$ ) laser is controlled by a feedback algorithm to sustain a predefined number of localizations per frame. Typical acquisition parameters can be found in Table 4 . Coverslips containing prepared samples were placed into a custom build sample holder and $500 \mu \mathrm{L}$ of suitable buffer, depending on the used cell line and experiment (Table 5), was added. To avoid a pH drift caused by accumulation of glucuronic acid in GLOX-buffers, the 
buffer solution was exchanged after about $2 \mathrm{~h}$ of imaging. Samples were imaged until close to all fluorophores were bleached and no further localizations were detected under continuous UV irradiation.

Pixel size calibration-The effective pixel size of the microscope was calibrated by translating fluorescent beads, immobilized on a coverslip, with a calibrated sample stage (SmarAct, Oldenburg, Germany) that operated in close loop. From the measured translation of many beads the pixel size could be calibrated with a high accuracy.

Widefield, SIM and SRRF on Expanded Samples-After expansion (protocol described above) U2OS-Nup96-mEGFP cells labeled with an Atto488-coupled anti-GFP nanobody were imaged in a Zeiss Elyra PS.1 system. An 100x TIRF objective (PlanAPOCHROMAT $100 / 1.46$ Oil, Zeiss) was used, with additional 1.6 magnification, to collect fluorescence onto an EMCCD camera (iXon Ultra 897, Andor), yielding a pixel size of $100 \mathrm{~nm}$. Sample was illuminated with a $488 \mathrm{~nm}$ laser set at $150 \mathrm{~mW} / \mathrm{cm}^{2}$. Widefield images were collected with $100 \mathrm{~ms}$ exposure, SIM images with $100 \mathrm{~ms}$ exposure and 5 grid roations, each SRRF image was generated from a frame-burst of 100 images acquired at 33 Hz. SIM reconstructions were generated with the Zeiss Elyra Zen software using automatic settings. SRRF images were analysed with NanoJ-SRRF ${ }^{24}$ using standard settings. Images were validated for quality using SIMCheck ${ }^{6}$ (SIM) and NanoJ-SQUIRREL ${ }^{7}$ (SIM and SRRF).

Confocal microscopy-Fixed U2OS-Nup96-mEGFP samples on $35 \mathrm{~mm}$ glass bottom dishes were prepared according to the preparation protocol described above and imaged using an Olympus FV3000 laser scanning microscope. A 60x / 1.40 NA oil immersion objective (Olympus; PLAPON 60XOSC2) was used in combination with a motorized stage, operated by the Fluoview software (Olympus). Pixel size was set to $\sim 70 \mathrm{~nm}$ in $\mathrm{x}$ and $\mathrm{y}$. Fluorescence emission went through a 550/100 bandpass filter and a 1.0 airy unit $(202 \mu \mathrm{m})$ wide pinhole before detection on $4 \mathrm{GaAsP}$ spectral detectors. For each nucleus, a z-stack, consisting of 3-5 planes $250 \mathrm{~nm}$ apart from each other, was acquired around the basal plane of the nucleus to obtain maximum fluorescence intensity for all NPCs.

Airy-scan microscopy-35 mm glass bottom dishes containing U2OS-Nup96-mEGFP were fixed in accordance to the previously described protocol. A Zeiss LSM 880 with an additional Airy FAST detector module (Zeiss) was used for airy-image acquisition in combination with a $63 \mathrm{x} / 1.4$ NA oil immersion objective (Zeiss; Plan-Apochromat 63x/1.4 Oil DIC M27). The system was operated by the ZEN software (Zeiss; black edition). Pixel size was set to $\sim 40 \mathrm{~nm}$ in $\mathrm{x}$ and $\mathrm{y}$ direction. Samples were focused on the basal plane of the nucleus and mEGFP was excited using a $488 \mathrm{~nm}$ laser. Emission was collected through a 495-550 nm bandpass filter, $570 \mathrm{~nm}$ longpass filter and a 1.25 airy unit $(\sim 0 \mu \mathrm{m})$ pinhole onto the $32 \mathrm{GaAsP}$ detector elements. A z-stack, consisting of 3-5 slices $200 \mathrm{~nm}$ apart from each other around the basal plane were acquired for each nucleus. Post-processing was done with ZENs airy-scan processing, using automatic deconvolution parameters.

STED microscopy-Samples were prepared according to the protocol for nanobody staining of U2OS-Nup96-GFP samples and were imaged on an Abberior STED/RESOLFT 
microscope (Abberior Instruments; Expert Line) running the Imspector software (Abberior Instruments). The microscope comprises of an IX83 stage (Olympus) in combination with a UPlan-S Apochromat 100x / NA 1.40 oil objective (Olympus). Pixel size was set to $15 \mathrm{~nm}$ in $\mathrm{x}, \mathrm{y}$ direction. Super-resolved images were acquired by donut-shaped depletion using a 775 $\mathrm{nm}$ pulsed laser along with a $640 \mathrm{~nm}$ pulsed laser, exciting STAR 635P tagged Nup96mEGFP. A single plane of the lower side of the nucleus was imaged. Emission was collected through a $685 / 70 \mathrm{~nm}$ bandpass filter. We used a depletion power of approx. $150 \mathrm{~mW}$ in the sample. Higher depletion powers could in principle increase the resolution further, but in our case lead to strong bleaching and high noise for these rather dim samples.

Ratiometric dual-color SMLM-For ratiometric dual-color imaging of AF647 and CF680, the emitted fluorescence was split by a 665LP beamsplitter (Cat\#ET665lp, Chroma), filtered by a $685 / 70$ (Cat\#ET685/70m, Chroma) bandpass filter (transmitted light) or a 676/37 (Cat\#FF01-676/37-25, Semrock) bandpass filter (reflected light) and imaged side by side on the EMCCD camera. The color of the individual blinks was assigned by calculating the ratio of the intensities in the two channels.

Astigmatic 3D SMLM-3D SMLM data was acquired using a cylindrical lens ( $\mathrm{f}=1000$ $\mathrm{mm}$; Cat\#LJ1516L1-A, Thorlabs) to introduce astigmatism. The data were fitted and analyzed as described previously ${ }^{61}$. First, z-stacks with known displacement of several (15-20) fields of view of TetraSpeck beads on a coverslip were acquired to generate a model of the experimental point spread function. This model was then used to determine the zposition of the individual localizations. To correct for depth-dependent aberrations, we acquired stacks of beads in agarose to determine the fitting errors as described previously ${ }^{30}$.

\section{Data analysis}

All data analysis was performed with custom software written in MATLAB and is available as open source (github.com/jries/SMAP). Installation instructions are found in the README.md, and step-by-step guides on how to use the software to perform all analyses used in this manuscript are available via the Help menu.

Fitting -2D data were fitted with a symmetric Gaussian PSF model (free fitting parameters: $x, y$, PSF size, photons per localization, background per pixel) using maximum likelihood estimation (MLE) ${ }^{62}$. Astigmatic 3D data were fitted with an experimentally derived PSF model (free fitting parameters: $\mathrm{x}, \mathrm{y}, \mathrm{z}$, photons per localization, background per pixel), also using an MLE fitter ${ }^{61}$.

Post-processing- $\mathrm{x}-, \mathrm{y}-$, and, when applicable, $\mathrm{z}-$ positions were corrected for residual drift by a custom algorithm based on redundant cross-correlation. Localizations persistent over consecutive frames (detected within $35 \mathrm{~nm}$ from one another and with a maximum gap of one dark frame) were merged into one localization by calculating the weighted average of $\mathrm{x}, \mathrm{y}$ and $\mathrm{z}$ positions and the sums of photons per localization and background. Localizations were filtered by the localization precision ( $0-10 \mathrm{~nm}$ for dSTORM; $0-25 \mathrm{~nm}$ for PALM) to exclude dim localizations, and for 2D data by the fitted size of the PSF ( $0-150 \mathrm{~nm}$ laterally) to exclude localizations that were strongly out-of-focus. Additionally, poorly fitted 
localizations were excluded if their log-likelihood (LL) was smaller than the mean(LL) - $3 *$ STD(LL). Super-resolution images were constructed with every localization rendered as a 2D elliptical Gaussian with a width proportional to the localization precision (factor 0.4). The reported mean photons per localization were calculated based on these merged and filtered localizations.

Determination of the expansion factor-To determine the local expansion factor from the Ex-SIM data set, we manually selected positions of 203 nuclear pores and fitted a cropped image of the pore with a model that consisted of a ring convolved with a Gaussian function, treating the radius and the standard deviation of the Gaussian as free fitting parameters. We then re-fitted the data keeping the standard deviation of the Gaussian fixed to its mean value. By comparing the mean value of the radius with that one measured on a calibrated SMLM microscope, we directly determined the expansion factor.

Segmentation-To automatically segment nuclear pore complexes, we convolved the reconstructed superresolution image with a kernel consisting of a ring with a radius corresponding to the radius of the NPC, convolved with a Gaussian. Local maxima over a user-defined threshold were treated as candidate NPCs. These candidates included many aberrant structures. We cleaned up the segmentation by a two-step filtering process: 1) We fitted the localizations corresponding to each candidate with a circle to reject structures with very small (typically $<40 \mathrm{~nm}$ ) or very large $(>70 \mathrm{~nm})$ radii. 2) We re-fitted the localizations with a circle of fixed radius to determine its center coordinates, and rejected structures where more than $25 \%$ of the localizations were within $40 \mathrm{~nm}$ of the center (structures that visually did not resemble NPCs) or more than $40 \%$ of the localizations were further away than $70 \mathrm{~nm}$ (structures that were usually composed of two adjacent NPCs and wrongfully segmented).

We segmented many data sets manually and compared that segmentation with the automatic segmentation and found an excellent agreement with less than $1.2 \%$ difference in measured ELE values and less than $5 \%$ error in the mean number of localizations per NPC.

Geometric analysis-All geometric analysis was performed on NPCs segmented as described above, based on the coordinates of the localizations.

Analysis of profiles. Profiles in the $x-y$ plane were constructed by 1) selecting a linear ROI in the direction the profile is calculated, 2) selecting only localizations in a rectangular ROI along the line profile and with a given width, 3) rotating the coordinates such that the $x$ '-axis is along the direction of the line profile, 4) calculating a histogram of the $\mathrm{x}$ ' coordinates.

This histogram was then fitted with a single or double Gaussian function. For profiles along the z-direction we 1) defined a ROI, 2) calculated the histogram of z-coordinates for localizations within this ROI and 3 ) fitted the histogram with a single or double Gaussian function.

We want to stress that care must be taken that profiles are constructed from a sufficient number of localizations, and are never measured in a superresolution image where localizations are rendered with a Gaussian kernel. Otherwise even single localizations can 
result in 'profiles' with arbitrary small width and two random localizations can be 'resolved' if their distance is larger than the arbitrary kernel size. This holds true for any profile analysis of SMLM data and is not restricted to NPCs.

Radius of the NPC. The radius of the circular NPC structures was determined by directly fitting the coordinates of the localizations with a circular model treating the $\mathrm{x}$ and $\mathrm{y}$ coordinates and the radius as free fitting parameters.

Distance between cytoplasmic and nucleoplasmic rings in 2D data. Ring distances were measured on 2D data sets where the focus was set to the mid-plane of the nucleus. 1) We manually segmented structures on vertical parts of the nuclear envelope. 2) We constructed profiles perpendicular to the nuclear envelope with a width of $200 \mathrm{~nm}$ by calculating the histogram of rotated localizations. 3) We fitted the profiles with a double-Gaussian function to determine the distance of the rings.

Distance of cytoplasmic and nucleoplasmic rings from 3D data. Segmented localizations were fitted in 3D with a template describing two parallel rings with a fixed radius (mean of the radius as measured before) and variable $\mathrm{x}, \mathrm{y}$ and $\mathrm{z}$ positions, rotation angles and distance between the rings. As a validation, we used the fitted rotation angles to rotate the localizations so that all NPCs were aligned and fitted the z-profile with a double Gaussian as described above for 2D data.

Azimuthal angle. We determined the azimuthal angle between the cytoplasmic and nucleoplasmic rings from 3D data. 1) we fitted the localizations with a circle to determine its $\mathrm{x}$ and $\mathrm{y}$ center coordinates. 2) We determined the axial position of the NPC by fitting the $\mathrm{z}-$ profile with a double-Gaussian as described above. 3) We separated localizations belonging to the upper and lower ring. 4) We transformed the $\mathrm{x}$, y coordinates to polar coordinates. 5) We constructed histograms of the polar angles. 6) we calculated the auto- and crosscorrelation curves of these histograms taking into account the circular boundary conditions. 7) We calculated the average correlation curves for all NPCs. 8) We fitted the average crosscorrelation curve with a cosine function of fixed frequency and varying phase. We fitted the offset and amplitude of the trigonometric function by $3^{\text {rd }}$-degree polynomials. We excluded the central $24^{\circ}$ from the fit as they contained strong contributions from the re-activation of fluorophores. 9) The azimuthal angle corresponds to the fitted phase of the trigonometric function.

Determination of effective labeling efficiencies-To count the number of visible corners in each nuclear pore complex we used the following approach: 1) The segmented and filtered localizations were fitted by a circle of fixed radius corresponding to the mean radius as determined before and coordinates were converted into polar coordinates $\phi_{i}, r_{i}$. 2) Localizations too close to the center of the ring $\left(r_{i}<30 \mathrm{~nm}\right)$ or too far away $\left(r_{i}>70 \mathrm{~nm}\right)$ were excluded as background localizations. 3) We determined the rotation of the structure by minimizing

$$
\phi_{\text {rot }}=\arg \min _{\phi_{\text {rot }}}\left(\phi_{\text {rot }}-\phi_{i} \bmod \pi / 4\right)
$$


4) we counted the number of segments containing a localization from a histogram of $\phi_{i}$ of with a bin width of $\pi / 4$ and a start bin of $\phi_{r o t}-\pi / 8.5$ ) We constructed a histogram of the number of corners of all NPCs in the data set and fitted it using the probabilistic model as described below, using the effective labeling efficiency as a free fitting parameter. 6) To calculate the statistical error, we used bootstrapping with typically 20 re-sampled data sets.

Probabilistic model for effective labeling efficiency. The binomial probability density function

$$
B(k \mid n, p)=\left(\begin{array}{l}
n \\
k
\end{array}\right) p^{k}(1-p)^{n-k}
$$

describes the probability of observing $k$ successes in $n$ independent trials, where the probability of success in any given trial is $p$. Thus, the probability of a corner of the NPC (consisting of 4 labels) to be dark is $p_{\text {dark }}=B\left(0 \mid 4, p_{\text {label }}\right)$ and the probability to see a corner with at least one label is $p_{\text {bright }}=1-p_{\text {dark }}$. The probability of $N$ out of 8 corners being bright and visible is:

$$
p\left(N \mid p_{\text {label }}\right)=B\left(N \mid 8, p_{\text {bright }}\right)=B\left(N \mid 8,1-B\left(0 \mid 4, p_{\text {label }}\right)\right)
$$

Determination of number of localizations per fluorophore. The number of localizations (blinking events) $N_{b}$ that are detected per fluorophore can be directly calculated from the ELE, the number of localizations per NPC $N_{l}$ and the number of Nup96 molecules per NPC $N_{\text {Nup96 }}=32$ :

$$
N_{b}=\frac{N_{l}}{N_{\mathrm{Nup} 96} E L E}
$$

Simulations-To validate our analysis routines, we performed realistic simulations based on a two-state (bright and dark) fluorophore model with bleaching ${ }^{63}: 1$ ) We defined the 3D coordinates of the 32 Nup96 proteins in the nuclear pore complex based on our calibration (Figure 2). 2) we randomly displace all coordinates by a random vector and rotate the coordinates in 3D by random angles. 2) With a probability $p_{\text {label }}$ a protein is labeled and creates a localization. 3) A labeled protein has a probability $p_{\text {react }}$ to be reactivated. 4) Whenever a fluorophore is activated it appears at random during a frame and lives for $t_{I}$ frames, determined as a random variable from an exponential distribution. 5) When it is on, a fluorophore has a constant brightness. 7) The emitted photons in each frame are determined as a random Poisson variable with a mean corresponding to the average brightness during the frame. 8) For each frame we calculate the CRLB in $\mathrm{x}, \mathrm{y}$ and $\mathrm{z}$ from the number of photons and the background ${ }^{64}$. 9) This error is added to the true $x, y$ and $z$ positions of the fluorophores as normally distributed random values with a variance corresponding to the respective calculated CRLB.

The simulated localizations were processed with the same data analysis pipeline as the real data. 
Counting of protein copy numbers - Counting in diffraction-limited microscopy using Nup96-mEGFP as a reference. We used a simple data analysis procedure to compare the brightness of reference and target structures in confocal images: 1) We subtracted the image offset, and if required corrected the images for photobleaching. 2) We calculated the maximum intensity projection of 3 frames around the focal plane of the nuclear pore structures and convolved the image with a Gaussian ( $\sigma=0.5$ pixels). 3) We up-sampled the image by a factor of two using cubic spline interpolation. 4) We determined all local maxima and chose a threshold based on the histogram of intensity values of those maxima. 5) We fitted the histogram of maxima intensities above the threshold with a Gaussian function to determine a robust estimate of the mean of the intensity values $\left\langle I_{t}\right\rangle$ and $\left\langle I_{I}\right\rangle$ for reference and target cell lines. 6) With $N_{r}$ copies of the reference protein in the complex, the copy number in the target complex is then $N_{t}=N_{r}\left\langle I_{t}\right\rangle /\left\langle I_{r}\right\rangle$.

Counting in mammalian cells using Nup96-mMaple as a reference. 1) We automatically segmented reference and target data as described above and only considered nuclear pores in the focus (mean value of the fitted size of the PSF smaller than $145 \mathrm{~nm}$ ). 2) We counted the number of merged localizations $\left(L_{F}, L_{t}\right)$ in a circular ROI of a diameter of $220 \mathrm{~nm}$. 3) From the mean number of localizations per nuclear pore complex $\left\langle L_{t}\right\rangle$ and $\left\langle L_{r}\right\rangle$ we can calculate the copy number of the target complex $N_{t}=N_{r}\left\langle L_{t}\right\rangle /\left\langle L_{r}\right\rangle$.

Counting in yeast cells using Nup188 as a reference. 1) We manually segmented NPCs in yeast cells and excluded structures that were out-of-focus, at the edge of the nucleus or too close to other structures. 2) Based on the intensity of Abp1-mEGFP in a diffraction limited channel we assigned all NPCs in a cell to belong to the reference cell line (significant mEGFP signal) or to the target cell line (no mEGFP signal). 3) We determined the number of localizations in a circular ROI of a diameter of $150 \mathrm{~nm}$. 4) As above, we determined the mean number of merged localizations and from those the copy number of the target complex.

Model to estimate steady state maturation fraction. Here we derive a very simple model to estimate the fraction of matured photoconvertible fluorescent protein (e.g. mMaple) in the steady state neglecting degradation. $P$ denotes the amount of not yet matured protein, $M$ the amount of the matured protein and $k_{m}$ is the maturation rate. We assume exponential growth (growth rate $k_{g}$ ) of the organism and thus of the proteins:

$$
\frac{d P}{d t}=k_{g}(P+M)-k_{m} P, \quad \frac{d M}{d T}=k_{m} P
$$

The solution is:

$$
\frac{P}{P+M}=\frac{e^{-\left(k_{m}+k_{g}\right) t_{k}+k_{g}}}{k_{m}+k_{g}} \underset{t \rightarrow \infty}{\longrightarrow} \frac{k_{g}}{k_{m}+k_{g}}
$$

Assuming a doubling time for yeast of $120 \mathrm{~min}$ and a maturation time for mMaple of $48 \mathrm{~min}$ we find that in the steady state on average $28 \%$ of the mMaple is not yet matured. For mammalian cells (generation time 1 day) the fraction is reduced to $3.2 \%$. 


\section{Supplementary Material}

Refer to Web version on PubMed Central for supplementary material.

\section{Acknowledgements}

PA-JF549 and Halo-Cy5 were a kind gift of Luke Lavis, HHMI Janelia Research Campus. We thank the EMBL advanced light microscopy facility (ALMF) for their help. This work was supported by the European Research Council (ERC CoG-724489, J.R., M.M., P.H., J.V.T.), the National Institutes of Health Common Fund 4D Nucleome Program (Grant U01 EB021223 / U01 DA047728 to J.E. and J.R.), the Allen Distinguished Investigator Program through The Paul G. Allen Frontiers Group (J.E.), the UK Biotechnology and Biological Sciences Research Council (BB/M022374/1; BB/P027431/1; BB/R000697/1; BB/S507532/1, R.H. and P.M.P.), the Wellcome Trust (203276/Z/16/Z, R.H. and P.M.P.), the EMBL Interdisciplinary Postdoc Programme (EIPOD) under Marie Curie Actions COFUND (Y.L.), the Human Frontier Science Program (RGY0065/2017 to J.R.) and the European Molecular Biology Laboratory (J.V.T., K.C., P.H., S.K.P., K.C.K., Y.L., Y.W., M.M., U.M., B.N., M.K., V.J.S., J.E. and J.R.). V.J.S. acknowledges support by the Boehringer Ingelheim Fonds.

\section{Data availability}

All processed data (lists of localizations) and for each condition at least one example file of raw data (camera frames of blinking fluorophores) are deposited on BioStudies (https:// www.ebi.ac.uk/biostudies/S-BSST257).

\section{Software availability}

All software is available at github.com/jries/SMAP.

\section{References}

1. Betzig E, et al. Imaging Intracellular Fluorescent Proteins at Nanometer Resolution. Science. 2006; 313:1642-1645. [PubMed: 16902090]

2. Rust MJ, Bates M, Zhuang X. Sub-diffraction-limit imaging by stochastic optical reconstruction microscopy (STORM). Nat Methods. 2006; 3:793-795. [PubMed: 16896339]

3. Xu K, Zhong G, Zhuang X. Actin, spectrin, and associated proteins form a periodic cytoskeletal structure in axons. Science. 2013; 339:452-456. [PubMed: 23239625]

4. Mund M, et al. Systematic Nanoscale Analysis of Endocytosis Links Efficient Vesicle Formation to Patterned Actin Nucleation. Cell. 2018; 174:884-896.e17. [PubMed: 30057119]

5. Szymborska A, et al. Nuclear Pore Scaffold Structure Analyzed by Super-Resolution Microscopy and Particle Averaging. Science. 2013; 341:655-658. [PubMed: 23845946]

6. Ball G, et al. SIMcheck: a Toolbox for Successful Super-resolution Structured Illumination Microscopy. Sci Rep. 2015; 5

7. Culley S, et al. Quantitative mapping and minimization of super-resolution optical imaging artifacts. Nat Methods. 2018; 15:263-266. [PubMed: 29457791]

8. Sage D, et al. Super-resolution fight club: assessment of 2D and 3D single-molecule localization microscopy software. Nat Methods. 2019; doi: 10.1038/s41592-019-0364-4

9. Steinhauer C, Jungmann R, Sobey TL, Simmel FC, Tinnefeld P. DNA Origami as a Nanoscopic Ruler for Super-Resolution Microscopy. Angew Chem Int Ed. 2009; 48:8870-8873.

10. Iinuma R, et al. Polyhedra self-assembled from DNA tripods and characterized with 3D DNAPAINT. Science. 2014; 344:65-69. [PubMed: 24625926]

11. Huang B, Jones SA, Brandenburg B, Zhuang X. Whole-cell 3D STORM reveals interactions between cellular structures with nanometer-scale resolution. Nat Methods. 2008; 5:1047-1052. [PubMed: 19029906]

12. Huang B, Wang W, Bates M, Zhuang X. Three-dimensional super-resolution imaging by stochastic optical reconstruction microscopy. Science. 2008; 319:810-813. [PubMed: 18174397] 
13. von Appen A, et al. In situ structural analysis of the human nuclear pore complex. Nature. 2015; 526:140-143. [PubMed: 26416747]

14. Durisic N, Laparra-Cuervo L, Sandoval Álvarez Á, Borbely JS, Lakadamyali M. Single-molecule evaluation of fluorescent protein photoactivation efficiency using an in vivo nanotemplate. Nat Methods. 2014; 11:156-162. [PubMed: 24390439]

15. Zanacchi FC, et al. A DNA origami platform for quantifying protein copy number in superresolution. Nat Methods. 2017; 14:789-792. [PubMed: 28650478]

16. Finan K, Raulf A, Heilemann M. A set of homo-oligomeric standards allows accurate protein counting. Angew Chem Int Ed Engl. 2015; 54:12049-12052. [PubMed: 26289028]

17. Zanacchi FC, Manzo C, Magrassi R, Derr ND, Lakadamyali M. Quantifying Protein Copy Number in Super-Resolution Using an Imaging-Invariant Calibration. Biophys J. 2019; doi: 10.1016/ j.bpj.2019.04.026

18. Löschberger A, et al. Super-resolution imaging visualizes the eightfold symmetry of gp210 proteins around the nuclear pore complex and resolves the central channel with nanometer resolution. J Cell Sci. 2012; 125:570-575. [PubMed: 22389396]

19. McEvoy AL, et al. mMaple: A Photoconvertible Fluorescent Protein for Use in Multiple Imaging Modalities. PLoS ONE. 2012; 7:e51314. [PubMed: 23240015]

20. Keppler A, Pick H, Arrivoli C, Vogel H, Johnsson K. Labeling of fusion proteins with synthetic fluorophores in live cells. Proc Natl Acad Sci U S A. 2004; 101:9955-9959. [PubMed: 15226507]

21. Los GV, et al. HaloTag: a novel protein labeling technology for cell imaging and protein analysis. ACS Chem Biol. 2008; 3:373-382. [PubMed: 18533659]

22. Klar TA, Jakobs S, Dyba M, Egner A, Hell SW. Fluorescence microscopy with diffraction resolution barrier broken by stimulated emission. Proc Natl Acad Sci U S A. 2000; 97:8206-8210. [PubMed: 10899992]

23. Chen F, Tillberg PW, Boyden ES. Expansion microscopy. Science. 2015; 347:543-548. [PubMed: 25592419]

24. Gustafsson N, et al. Fast live-cell conventional fluorophore nanoscopy with ImageJ through superresolution radial fluctuations. Nat Commun. 2016; 7

25. Pesce L, Cozzolino M, Lanzanò L, Diaspro A, Bianchini P. Measuring expansion from macro- to nanoscale using NPC as intrinsic reporter. J Biophotonics. 0

26. Gambarotto D, et al. Imaging cellular ultrastructures using expansion microscopy (U-ExM). Nat Methods. 2019; 16:71. [PubMed: 30559430]

27. Demmerle J, Wegel E, Schermelleh L, Dobbie IM. Assessing resolution in super-resolution imaging. Methods. 2015; 88:3-10. [PubMed: 26164701]

28. Nieuwenhuizen RPJ, et al. Measuring image resolution in optical nanoscopy. Nat Methods. 2013; 10:557-562. [PubMed: 23624665]

29. Schlichthaerle T, et al. Direct visualization of single nuclear pore complex proteins using genetically-encoded probes for DNA-PAINT. Angew Chem Int Ed. 2019; doi: 10.1002/ anie. 201905685

30. Li Y, Wu Y-L, Hoess P, Mund M, Ries J. Depth-dependent PSF calibration and aberration correction for 3D single-molecule localization. bioRxiv. 2019; doi: 10.1101/555730

31. Fricke F, Beaudouin J, Eils R, Heilemann M. One, two or three? Probing the stoichiometry of membrane proteins by single-molecule localization microscopy. Sci Rep. 2015; 5

32. Ries J, Kaplan C, Platonova E, Eghlidi H, Ewers H. A simple, versatile method for GFP-based super-resolution microscopy via nanobodies. Nat Methods. 2012; 9:582-584. [PubMed: 22543348]

33. Grimm JB, et al. Bright photoactivatable fluorophores for single-molecule imaging. Nat Methods. 2016; 13:985-988. [PubMed: 27776112]

34. Dempsey GT, Vaughan JC, Chen KH, Bates M, Zhuang X. Evaluation of fluorophores for optimal performance in localization-based super-resolution imaging. Nat Methods. 2011; 8:1027-1036. [PubMed: 22056676]

35. Hartwich TM, et al. A stable, high refractive index, switching buffer for super-resolution imaging. bioRxiv. 2018; doi: 10.1101/465492 
36. Klehs K, et al. Increasing the Brightness of Cyanine Fluorophores for Single-Molecule and Superresolution Imaging. ChemPhysChem. 2014; 15:637-641. [PubMed: 24376142]

37. Ong WQ, Citron YR, Schnitzbauer J, Kamiyama D, Huang B. Heavy water: a simple solution to increasing the brightness of fluorescent proteins in super-resolution imaging. Chem Commun Camb Engl. 2015; 51:13451-13453.

38. Ori A, et al. Cell type-specific nuclear pores: a case in point for context-dependent stoichiometry of molecular machines. Mol Syst Biol. 2013; 9:648-648. [PubMed: 23511206]

39. Otsuka $S$, et al. Nuclear pore assembly proceeds by an inside-out extrusion of the nuclear envelope. eLife. 2016; 5

40. Annibale P, Vanni S, Scarselli M, Rothlisberger U, Radenovic A. Quantitative photo activated localization microscopy: unraveling the effects of photoblinking. PLoS ONE. 2011; 6:e22678. [PubMed: 21818365]

41. Lee S-H, Shin JY, Lee A, Bustamante C. Counting single photoactivatable fluorescent molecules by photoactivated localization microscopy (PALM). Proc Natl Acad Sci. 2012; 109:17436-17441. [PubMed: 23045631]

42. Rollins GC, Shin JY, Bustamante C, Pressé S. Stochastic approach to the molecular counting problem in superresolution microscopy. Proc Natl Acad Sci U S A. 2014; 112:E110-E118. [PubMed: 25535361]

43. Puchner EM, Walter JM, Kasper R, Huang B, Lim WA. Counting molecules in single organelles with superresolution microscopy allows tracking of the endosome maturation trajectory. Proc Natl Acad Sci U S A. 2013; 110:16015-16020. [PubMed: 24043832]

44. Baldering TN, et al. Synthetic and genetic dimers as quantification ruler for single-molecule counting with PALM. Mol Biol Cell. 2019; 30:1369-1376. [PubMed: 30969885]

45. Kim SJ, et al. Integrative structure and functional anatomy of a nuclear pore complex. Nature. 2018; 555:475-482. [PubMed: 29539637]

46. Rajoo S, Vallotton P, Onischenko E, Weis K. Stoichiometry and compositional plasticity of the yeast nuclear pore complex revealed by quantitative fluorescence microscopy. Proc Natl Acad Sci U S A. 2018; 115:E3969-E3977. [PubMed: 29632211]

47. Wang S, Moffitt JR, Dempsey GT, Xie XS, Zhuang X. Characterization and development of photoactivatable fluorescent proteins for single-molecule-based superresolution imaging. Proc Natl Acad Sci U S A. 2014; 111:8452-8457. [PubMed: 24912163]

48. Beck M, Lučić V, Förster F, Baumeister W, Medalia O. Snapshots of nuclear pore complexes in action captured by cryo-electron tomography. Nature. 2007; 449:611-615. [PubMed: 17851530]

49. Deschamps J, Mund M, Ries J. 3D superresolution microscopy by supercritical angle detection. Opt Express. 2014; 22:29081-29091. [PubMed: 25402146]

50. Erdmann RS, et al. Labeling Strategies Matter for Super-Resolution Microscopy: A Comparison between HaloTags and SNAP-tags. Cell Chem Biol. 2019; doi: 10.1016/j.chembiol.2019.01.003

51. Koch B, et al. Generation and validation of homozygous fluorescent knock-in cells using CRISPRCas9 genome editing. Nat Protoc. 2018; 13:1465-1487. [PubMed: 29844520]

52. Sun X, et al. Development of SNAP-Tag Fluorogenic Probes for Wash-Free Fluorescence Imaging. ChemBioChem. 2011; 12:2217-2226. [PubMed: 21793150]

53. Pleiner T, et al. Nanobodies: site-specific labeling for super-resolution imaging, rapid epitopemapping and native protein complex isolation. eLife. 2015; 4:e11349. [PubMed: 26633879]

54. Göttfert F, et al. Strong signal increase in STED fluorescence microscopy by imaging regions of subdiffraction extent. Proc Natl Acad Sci U S A. 2017; 114:2125-2130. [PubMed: 28193881]

55. Tillberg PW, et al. Protein-retention expansion microscopy of cells and tissues labeled using standard fluorescent proteins and antibodies. Nat Biotechnol. 2016; 34:987-992. [PubMed: 27376584]

56. Mund M, Kaplan C, Ries J. Localization microscopy in yeast. Methods Cell Biol. 2014; 123:253271. [PubMed: 24974032]

57. Khmelinskii A, Meurer M, Duishoev N, Delhomme N, Knop M. Seamless Gene Tagging by Endonuclease-Driven Homologous Recombination. PLoS ONE. 2011; 6:e23794. [PubMed: 21915245] 
58. Deschamps J, Rowald A, Ries J. Efficient homogeneous illumination and optical sectioning for quantitative single-molecule localization microscopy. Opt Express. 2016; 24:28080-28090. [PubMed: 27906373]

59. Heilemann M, Margeat E, Kasper R, Sauer M, Tinnefeld P. Carbocyanine dyes as efficient reversible single-molecule optical switch. J Am Chem Soc. 2005; 127:3801-3806. [PubMed: 15771514]

60. Bates M, Blosser T, Zhuang X. Short-Range Spectroscopic Ruler Based on a Single-Molecule Optical Switch. Phys Rev Lett. 2005; 94:108101. [PubMed: 15783528]

61. Li Y, et al. Real-time 3D single-molecule localization using experimental point spread functions. Nat Methods. 2018; 15:367-369. [PubMed: 29630062]

62. Smith CS, Joseph N, Rieger B, Lidke KA. Fast, single-molecule localization that achieves theoretically minimum uncertainty. Nat Methods. 2010; 7:373-375. [PubMed: 20364146]

63. Sage D, et al. Super-resolution fight club: A broad assessment of 2D \& 3D single-molecule localization microscopy software. bioRxiv. 2018; doi: 10.1101/362517

64. Mortensen KI, Churchman LS, Spudich JA, Flyvbjerg H. Optimized localization analysis for single-molecule tracking and super-resolution microscopy. Nat Methods. 2010; 7:377-381. [PubMed: 20364147] 


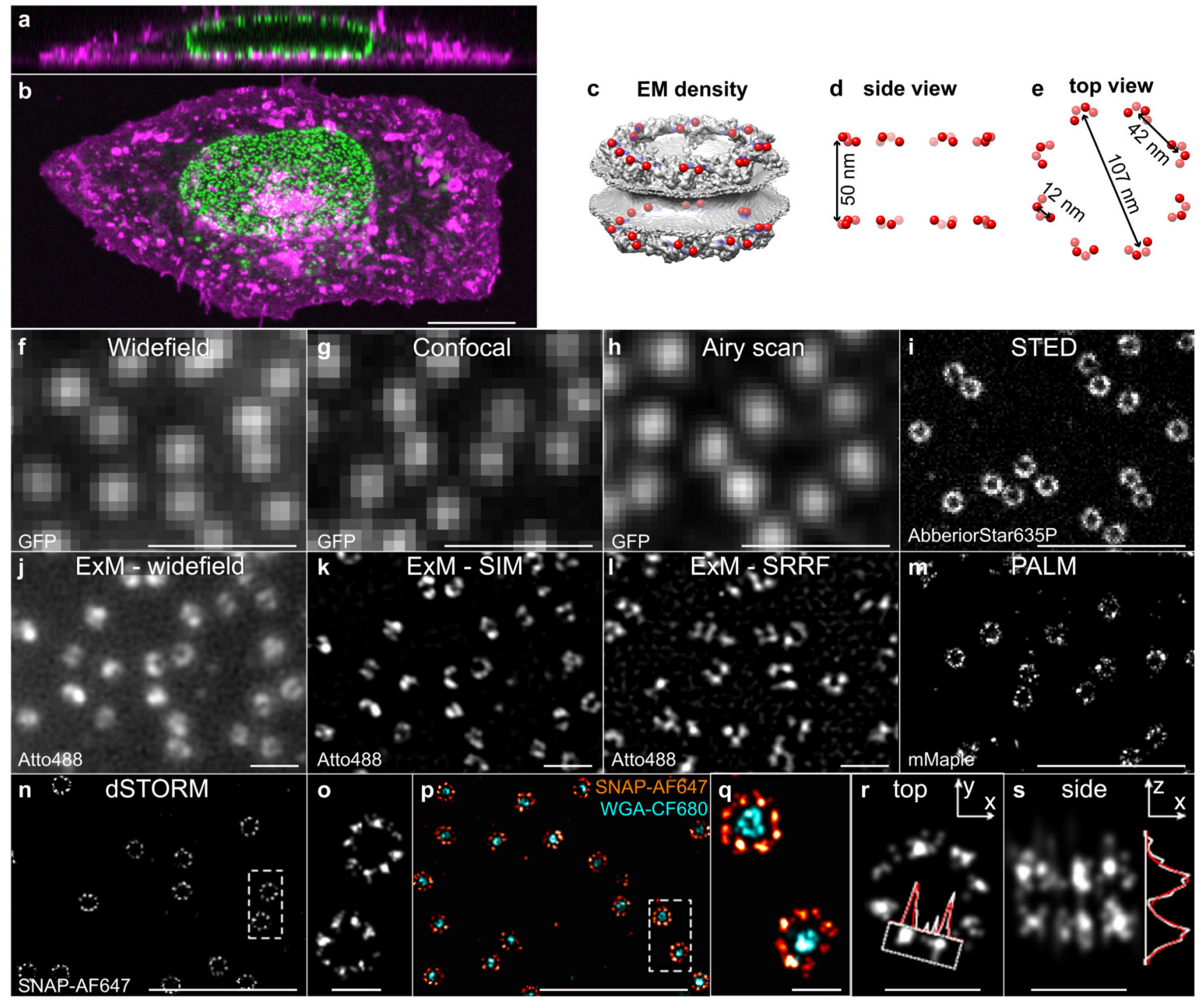

Figure 1. Nup96 cell lines.

(a) Representative confocal $x-z$ and (b) $x-y$ image of the Nup96-GFP cell line. Green: Nup96-GFP, magenta: membranes (DiD). (c) EM density of the nuclear pore complex ${ }^{13}$ with C-termini of Nup96 indicated in red. (d) Side view and (e) top view schematic. (f) Widefield, (g) confocal and (h) airy scan images of Nup96-GFP. (i) Raw STED image of Nup96-GFP labeled with an AberriorStar635P-coupled anti-GFP nanobody. Resolution estimates based on Fourier power spectra for $\mathbf{f}-\mathbf{i}$ can be found in Supplementary Figure 3 a. (j) Widefield expansion microscopy image of Nup96-GFP labeled with an Atto488-coupled anti-GFP nanobody. (k) As before, but imaged using structured illumination. Estimates of the expansion factor based on the analysis of the ring diameters can be found in Supplementary Figure 3c. (l) As before, but imaged using SRRF. (m) SMLM image of Nup96-mMaple, (n, o) SMLM of Nup96-SNAP labeled with BG-AF647 in GLOX/MEA. (p, q) Dual-color SMLM image of Nup96-SNAP labeled with BG-AF647 (red) and WGACF680 (cyan) in GLOX/MEA. (r, s) Corners of the NPC can be used as a resolution target in 
$\mathrm{x}, \mathrm{y}(\mathbf{r})$ and $\mathrm{z}(\mathbf{s})$. Resolution estimates based on Fourier Ring Correlation for $\mathbf{m}-\mathbf{q}$ can be found in Supplementary Figure 3b. Representative images of one (j-l), two (a,b,i), three (p$\mathbf{s})$, four (f-h,n,o) or six (m) independent experiments are shown. Scale bars $10 \mu \mathrm{m}(\mathbf{b}), 1 \mu \mathrm{m}$ $(\mathbf{f}-\mathbf{n}, \mathbf{p})$ and $100 \mathrm{~nm}(\mathbf{o}, \mathbf{q}, \mathbf{r}, \mathbf{s})$. 

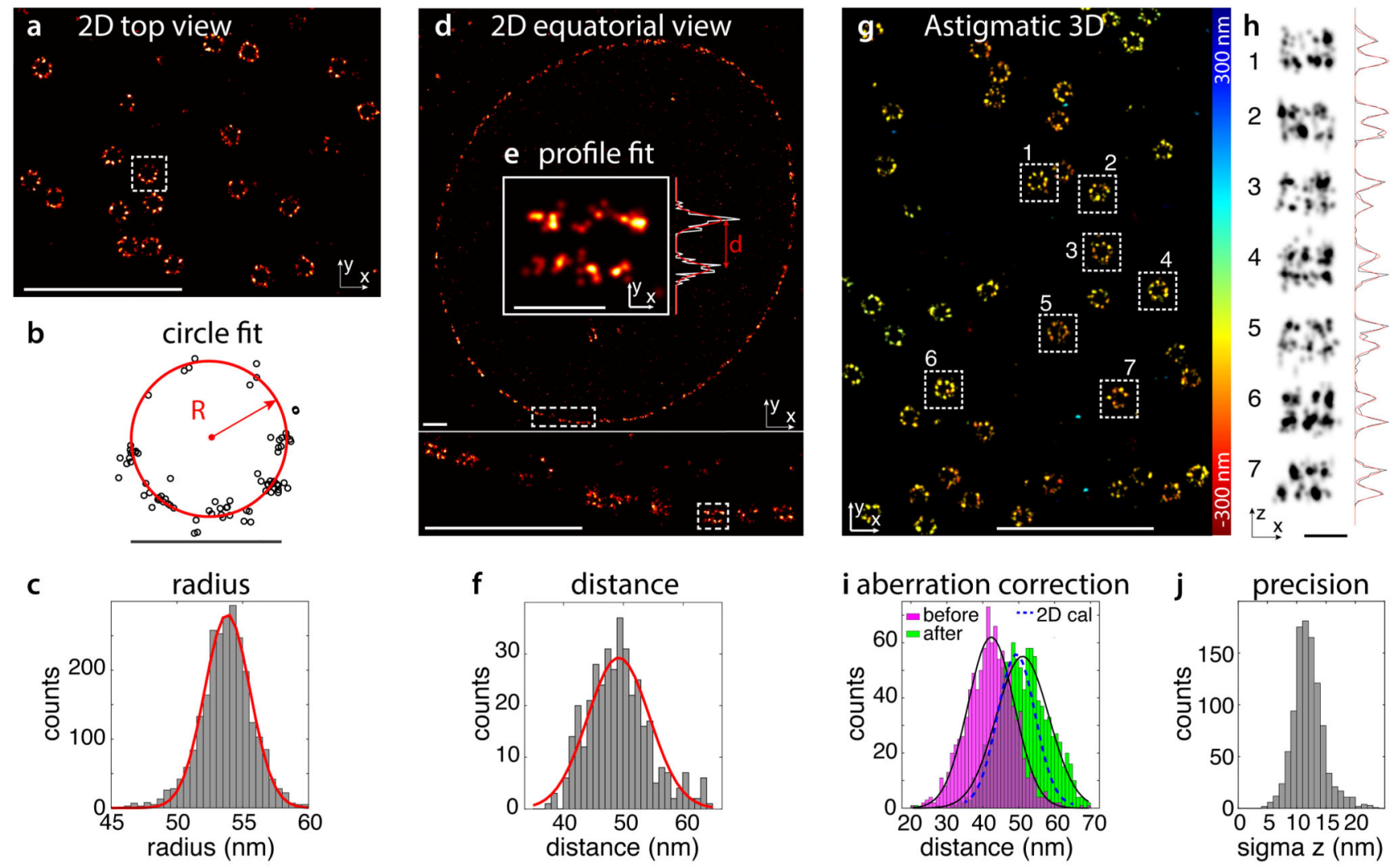

Figure 2. Nuclear pores as calibration reference standards. (a-h) experimental characterization of Nup-96 positions in the NPC. (a) SMLM image of lower nuclear envelope, (b) circle fit of a single NPC, (c) histogram of fitted radii ( $\mathrm{R}=$ $53.7 \mathrm{~nm} \pm 2.1 \mathrm{~nm}, \mathrm{~N}=3, \mathrm{n}_{\mathrm{C}}=7, \mathrm{n}_{\mathrm{NPC}}=2536$ ) (d) Equatorial SMLM image of Nup96, (e) a single NPC in a side view. A fit with a double Gaussian returns the ring-distance $d$ and the standard deviation of each ring. (f) Histogram of separation between rings $(\mathrm{d}=49.3 \pm 5.2$ $\mathrm{nm}, \mathrm{N}=2, \mathrm{n}_{\mathrm{C}}=14, \mathrm{n}_{\mathrm{NPC}}=379$ ). (g) 3D SMLM image of lower nuclear envelope. The localizations are color-coded according to their $\mathrm{z}$-position. (h) $\mathrm{X}-\mathrm{z}$ reconstructions with $\mathrm{z}$ profiles as indicated. (i) NPCs as calibration reference standard for astigmatic 3D

SMLM. Histogram of ring-distances before correction (magenta, $\mathrm{d}=42.1 \pm 1.1 \mathrm{~nm}, \mathrm{~N}=1$, $\left.\mathrm{n}_{\mathrm{C}}=3, \mathrm{n}_{\mathrm{NPC}}=1021\right)$ and after correcting for depth-induced calibration errors (green, $\mathrm{d}=$ $49.8 \pm 1.9 \mathrm{~nm}$ ). (j) Standard deviation of z-profiles from double Gaussian fit result in an upper bound for the experimental localization precision in $\mathrm{z}$ of $13.3 \pm 1.0 \mathrm{~nm}\left(\mathrm{~N}=1, \mathrm{n}_{\mathrm{C}}=3\right.$, $\left.\mathrm{n}_{\mathrm{NPC}}=1021\right)$. $\mathrm{N}$ denotes the number of biologically independent experiments, $\mathrm{n}_{\mathrm{C}}$ the number of imaged cells and $\mathrm{n}_{\mathrm{NPC}}$ the number of analyzed NPCs. All values depict weighted mean $\pm \mathrm{SD}$, based on $\mathrm{n}_{\mathrm{NPC}}$. Representative images of two (d,e) three (g) or four (a) independent experiments are shown. Scale bars $1 \mu \mathrm{m}(\mathbf{a}, \mathbf{d}, \mathbf{g}), 100 \mathrm{~nm}(\mathbf{b}, \mathbf{e}, \mathbf{h})$. All data on Nup96-SNAP-AF647 in GLOX/MEA. 


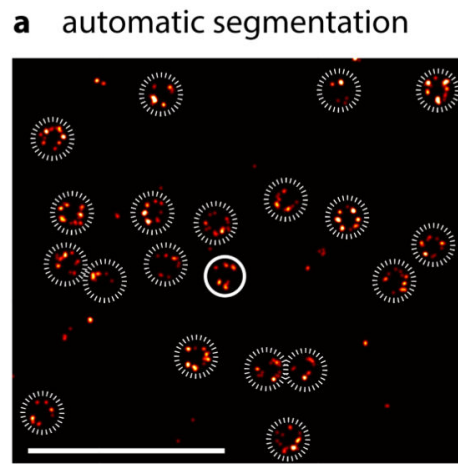

e Nup96-GFP nanobody-Q-AF647

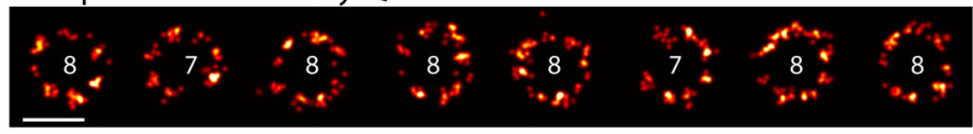

f Nup96-SNAP-AF647

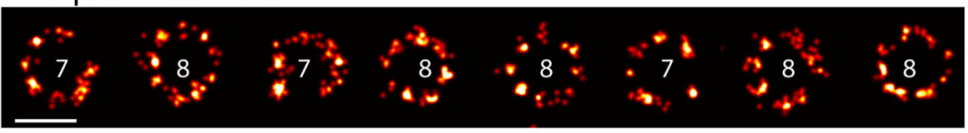

g Nup96-Halo-AF647

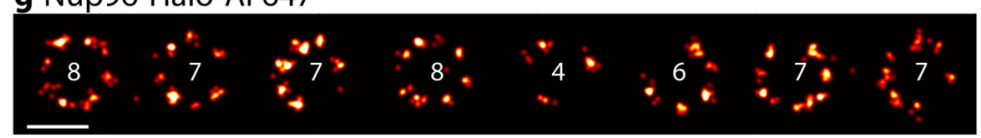

h Nup96-mMaple

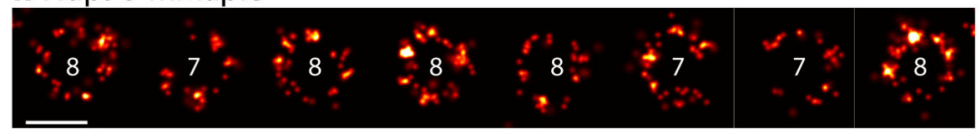

c rotational alignment, count corners

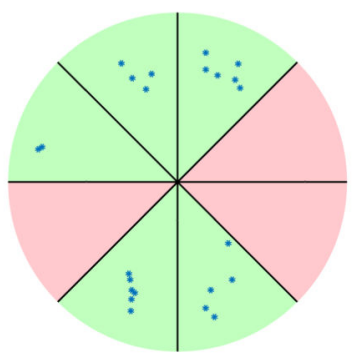

i GFP-NB-Q-AF647 GFP-NB-Q-CF680 GFP-NB-X4-AF647 GFP-NB-X4-CF680 GFP-NB-S-AF647** GFP-NB-S: $2 y$ old**

\begin{tabular}{|c|c|c|}
\hline GFP-Antibody & \multicolumn{2}{|c|}{ 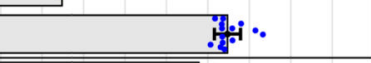 } \\
\hline SNAP-AF647 & \multicolumn{2}{|l|}{ मु. - } \\
\hline Halo-Cy5 & 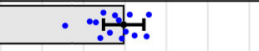 & \\
\hline Halo-O2-AF647 & : :-7: & \\
\hline Halo-O4-AF647 & itp & \\
\hline Halo-PAJF549* & (ajp. & \\
\hline mMaple & 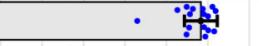 & \\
\hline mMaple live & t보. & \\
\hline & $40 \quad 60$ & $80 \quad 100$ \\
\hline
\end{tabular}

Figure 3. Effective labeling efficiencies.

(a-d) Workflow. (a) All NPCs in a cell are automatically segmented. (b) We fit a circle to the localizations and reject localizations outside a ring as background localizations. (c) We rotate the localizations to optimally fit an eightfold-symmetric template and count the number of slices that contain at least one localization. (d) We fit the histogram of the number of corners with a probabilistic model to directly obtain the absolute ELE. The statistical error is estimated by bootstrapping with 20 re-sampled data sets. (e-h) Gallery of NPCs. (e) Nup96-GFP labeled with an anti-GFP nanobody coupled to AF647. (f) Nup96-SNAP labeled with BG-AF647. (g) Nup96-Halo labeled with chloroalkane-AF647. (h) Nup96mMaple. The numbers indicate the numbers of visible corners the algorithm detected. (i) Effective labeling efficiencies for various cell lines and ligands. Bars denote the mean, error bars the standard deviation and individual data points measurements of a single cell. These data are derived from $\mathrm{N}$ biologically independent experiments, $\mathrm{n}_{\mathrm{C}}$ imaged cells and $\mathrm{n}_{\mathrm{NPC}}$ analyzed NPCs: GFP-NB-Q-AF647: $\mathrm{N}=2, \mathrm{n}_{\mathrm{C}}=6, \mathrm{n}_{\mathrm{NPC}}=2913$; GFP-NB-Q-CF680: $\mathrm{N}=2$, $\mathrm{n}_{\mathrm{C}}=5, \mathrm{n}_{\mathrm{NPC}}=1805 ;$ GFP-NB-X4-AF647: $\mathrm{N}=2, \mathrm{n}_{\mathrm{C}}=9, \mathrm{n}_{\mathrm{NPC}}=4303 ;$ GFP-NB-X4CF680: $\mathrm{N}=2, \mathrm{n}_{\mathrm{C}}=6, \mathrm{n}_{\mathrm{NPC}}=2011$; GFP-NB-S-AF647: $\mathrm{N}=2, \mathrm{n}_{\mathrm{C}}=4, \mathrm{n}_{\mathrm{NPC}}=8768$; GFPNB-S-AF647 (2y): $\mathrm{N}=2, \mathrm{n}_{\mathrm{C}}=3, \mathrm{n}_{\mathrm{NPC}}=1000 ;$ GFP-Antibody: $\mathrm{N}=3, \mathrm{n}_{\mathrm{C}}=14, \mathrm{n}_{\mathrm{NPC}}=$ 7380; SNAP-AF647: $\mathrm{N}=4, \mathrm{n}_{\mathrm{C}}=11, \mathrm{n}_{\mathrm{NPC}}=5372$; Halo-Cy5: $\mathrm{N}=5, \mathrm{n}_{\mathrm{C}}=14, \mathrm{n}_{\mathrm{NPC}}=5967$; 
Halo-O2-AF647: $\mathrm{N}=2, \mathrm{n}_{\mathrm{C}}=5, \mathrm{n}_{\mathrm{NPC}}=1393$; Halo-O4-AF647: $\mathrm{N}=2, \mathrm{n}_{\mathrm{C}}=6, \mathrm{n}_{\mathrm{NPC}}=3395$; Halo-PAJF549: $\mathrm{N}=3, \mathrm{n}_{\mathrm{C}}=17, \mathrm{n}_{\mathrm{NPC}}=4066$; mMaple: $\mathrm{N}=6, \mathrm{n}_{\mathrm{C}}=16, \mathrm{n}_{\mathrm{NPC}}=8146$; mMaple live: $\mathrm{N}=3, \mathrm{n}_{\mathrm{C}}=6, \mathrm{n}_{\mathrm{NPC}}=1343$; Example images for all labels can be found in Supplementary Figure 9, and imaging conditions are listed in Tables 4 and 5 (Methods). Representative images of two (e,g), four (a,f) or six (h) independent experiments are shown. Scale bars $1 \mu \mathrm{m}$ (a) and $100 \mathrm{~nm}(\mathbf{e}-\mathbf{h})$. *labeled in live cells, imaged after fixation.

**measured on Nup107-GFP. 


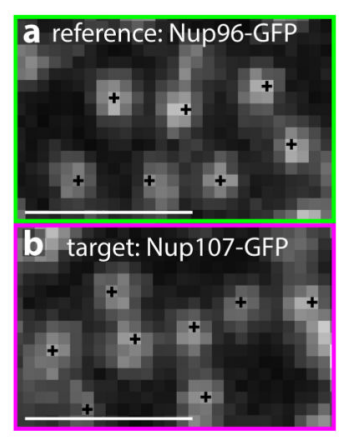

C brightness of GFP spots
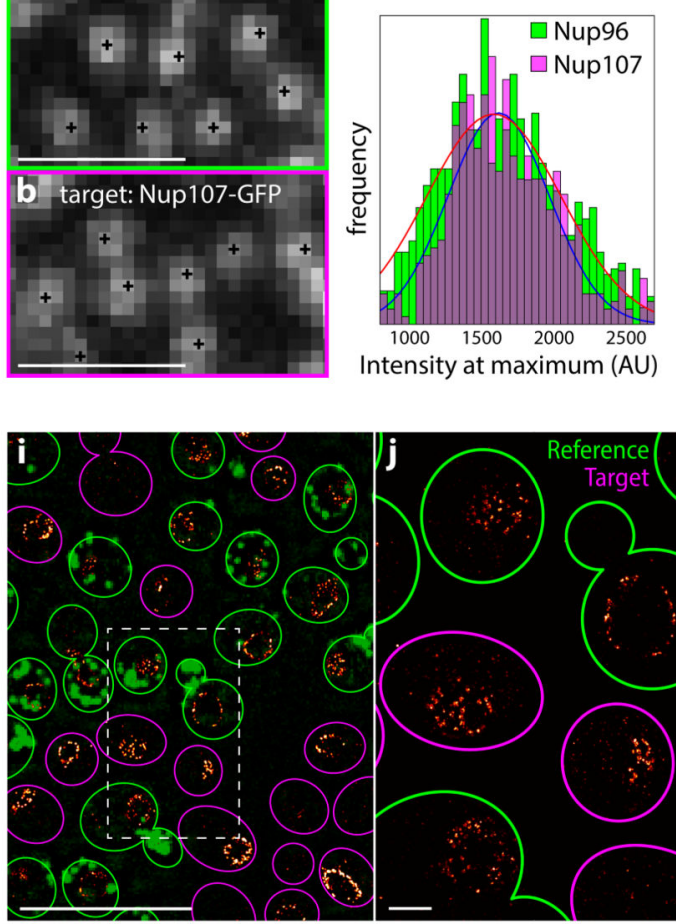
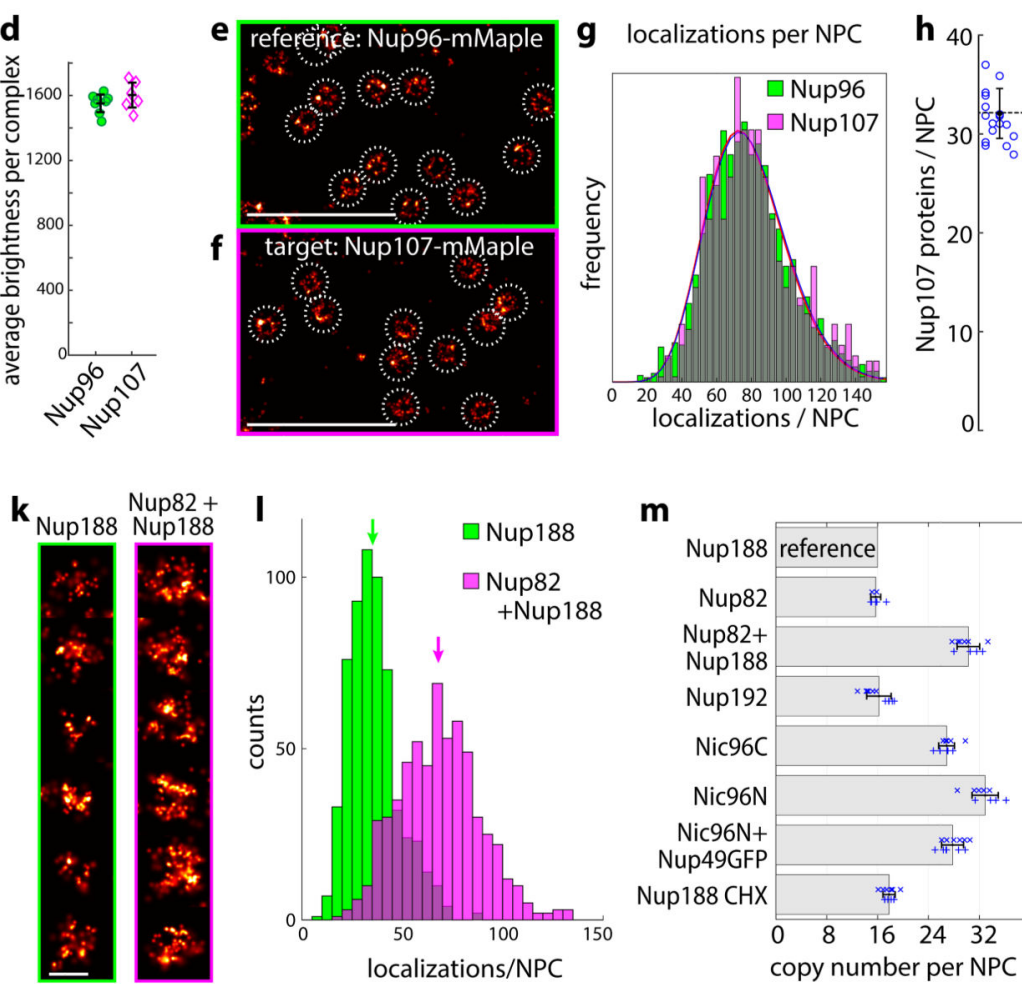

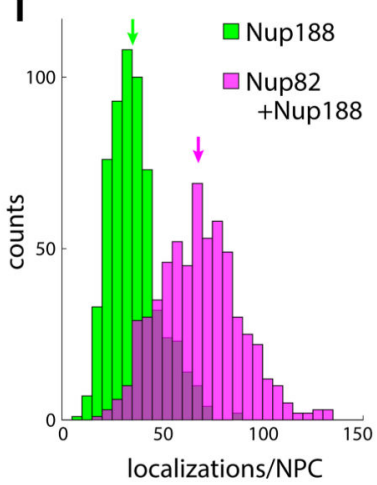

m

\begin{tabular}{|c|c|c|}
\hline Nup188 & reference & \\
\hline Nup82 & 单 & \\
\hline $\begin{array}{l}\text { Nup82+ } \\
\text { Nup188 }\end{array}$ & & 等 \\
\hline Nup192 & $x=$ & \\
\hline $\mathrm{Nic} 96 \mathrm{C}$ & & 栾 \\
\hline Nic96N & & $\times$ × \\
\hline $\begin{array}{r}\mathrm{Nic} 96 \mathrm{~N}+ \\
\text { Nup49GFP }\end{array}$ & & 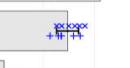 \\
\hline p188 CHX & & ex \\
\hline
\end{tabular}

Figure 4. Counting of protein copy numbers in complexes.

(a-d) Counting in diffraction limited microscopy. (a) Confocal image of the reference protein Nup96-GFP with the majority of nuclear pores resolved. (b) Confocal image of the target protein Nup107-GFP imaged with the same microscope settings. (c) Histograms of intensities of local maxima (see Methods) for the reference and target structures together with Gaussian fit to determine the mean intensity values. (d) Mean intensity values for several reference and target cells. These values show a small variation and are similar for reference $\left(\left\langle I_{\text {ref }}\right\rangle 1552=55 \mathrm{ADU}, \mathrm{N}=1, \mathrm{n}_{\mathrm{C}}=8, \mathrm{n}_{\mathrm{R}}=10104\right)$ and target complex $\left(\left\langle I_{\mathrm{tar}}\right\rangle=\right.$ $1603 \pm 77$ ADU, $\mathrm{N}=1, \mathrm{n}_{\mathrm{C}}=6, \mathrm{n}_{\mathrm{T}}=7178$ ). (e-h) Counting with SMLM. (e) Reconstructed superresolution image for reference cell line Nup96-mMaple and (f) for target cell line Nup107-mMaple. NPC structures are automatically segmented to determine the numbers of localizations per NPC. (g) Histogram of number of localizations per NPC for reference and target. The number of Nup107-mMaple proteins per NPC is calculated from the average relative number of localizations. (h) The stoichiometry of Nup107 in the NPC ( $n_{\text {Nup107 }}=$ $32.1 \pm 2.5, \mathrm{~N}=5, \mathrm{n}_{\mathrm{C}}=13, \mathrm{n}_{\mathrm{T}}=1928$ ) shows a high accuracy and low statistical errors of this counting approach. (i-m) Counting in yeast. (i) Mixture of Nup188-mMaple+Abp1GFP reference cell lines with Nup82-mMaple+Nup188-mMaple target cell lines, which can be distinguished by the GFP signal. (j) Superresolution reconstruction and (k) individual nuclear pores. (l) Histograms of the number of localizations per nuclear pore, arrows indicate the mean $\left(\mathrm{N}=2, \mathrm{n}_{\mathrm{C}}=508, \mathrm{n}_{\mathrm{NPC}}=1190\right.$ for Nup188 and $\mathrm{n}_{\mathrm{NPC}}=1176$ for Nup82+Nup188). (m) Copy number of several yeast nucleoporins per NPC, determined using Nup188 as a reference. These data are derived from: Nup82: $\mathrm{N}=2, \mathrm{n}_{\mathrm{C}}=242, \mathrm{n}_{\mathrm{T}}=$ $678, \mathrm{n}_{\mathrm{R}}=686 ;$ Nup82+Nup188: $\mathrm{N}=2, \mathrm{n}_{\mathrm{C}}=508, \mathrm{n}_{\mathrm{T}}=1176, \mathrm{n}_{\mathrm{R}}=1190 ;$ Nup192: $\mathrm{N}=2, \mathrm{n}_{\mathrm{C}}$ 
$=558, \mathrm{n}_{\mathrm{T}}=992, \mathrm{n}_{\mathrm{R}}=916 ; \operatorname{Nic} 96 \mathrm{C}: \mathrm{N}=2, \mathrm{n}_{\mathrm{C}}=304, \mathrm{n}_{\mathrm{T}}=1102, \mathrm{n}_{\mathrm{R}}=1127 ; \operatorname{Nic} 96 \mathrm{~N}: \mathrm{N}=2$, $\mathrm{n}_{\mathrm{C}}=532, \mathrm{n}_{\mathrm{T}}=1078, \mathrm{n}_{\mathrm{R}}=1079 ;$ Nic96N+Nup49GFP: $\mathrm{N}=2, \mathrm{n}_{\mathrm{C}}=303, \mathrm{n}_{\mathrm{T}}=1137, \mathrm{n}_{\mathrm{R}}=$ 1149; Nup188 (CHX treatment): $\mathrm{N}=2, \mathrm{n}_{\mathrm{C}}=521, \mathrm{n}_{\mathrm{T}}=1157, \mathrm{n}_{\mathrm{R}}=1154$. $\mathrm{N}$ denotes the number of biologically independent experiments, $\mathrm{n}_{\mathrm{C}}$ the number of analyzed cells, and $\mathrm{n}_{\mathrm{T}} / \mathrm{n}_{\mathrm{R}}$ the number of analyzed NPCs for the counting target/reference. Bars denote the mean, error bars the standard deviation and data points individual acquisitions. Shown values depict weighted mean $\pm \mathrm{SD}$, based on $\mathrm{n}_{\mathrm{NPC}}$. Representative images of one (b), two (a,i-k), five (f) or six (e) independent experiments are shown. Scale bars $10 \mu \mathrm{m}(\mathbf{i}), 1 \mu \mathrm{m}(\mathbf{a}, \mathbf{b}, \mathbf{e}, \mathbf{f}, \mathbf{j})$, $100 \mathrm{~nm}(\mathbf{k})$. 


\section{Table 1}

\section{Imaging conditions.}

Effective labeling efficiency, mean photons per localization and mean localizations per fluorophore for Nup96SNAP-AF647 and Nup96-mMaple in commonly used imaging buffers. Analysis performed after merging localizations occurring in consecutive frames (Methods). All values are weighted mean $\pm \mathrm{SD}$, based on number of analyzed NPCs. N denotes the number of biologically independent experiments. Example images can be found in Supplementary Figure 9.

\begin{tabular}{|c|c|c|c|c|c|}
\hline Sample & Buffers & Effective LE \% & $\begin{array}{l}\text { Photons per } \\
\text { localization }\end{array}$ & $\begin{array}{l}\text { Localizations per } \\
\text { fluorophore }\end{array}$ & $\begin{array}{c}\text { N / cells / NPCs } \\
\text { analyzed }\end{array}$ \\
\hline \multirow[t]{4}{*}{ SNAP-AF647 } & 35 mM MEA + GLOX & $58 \pm 3$ & $10168 \pm 982$ & $3.9 \pm 0.9$ & $4 / 11 / 5372$ \\
\hline & $\begin{array}{l}35 \mathrm{mM} \text { MEA + GLOX in } \\
\mathrm{D}_{2} \mathrm{O}\end{array}$ & $56 \pm 3$ & $10079 \pm 423$ & $3.6 \pm 0.4$ & $2 / 5 / 3379$ \\
\hline & 143 mM BME + GLOX & $64 \pm 4$ & $12904 \pm 689$ & $5.4 \pm 0.5$ & $3 / 8 / 3724$ \\
\hline & $\begin{array}{l}35 \mathrm{mM} \text { MEA }+50 \mathrm{mM} \\
\text { sodium sulfite }\end{array}$ & $40 \pm 2$ & $7006 \pm 513$ & $1.5 \pm 0.1$ & $2 / 5 / 2708$ \\
\hline mMaple fixed & $50 \mathrm{mM}$ Tris in $\mathrm{H}_{2} \mathrm{O}$ & $55 \pm 4$ & $1169 \pm 36$ & $2.5 \pm 0.2$ & $2 / 7 / 3126$ \\
\hline mMaple fixed & $50 \mathrm{mM}$ Tris in $\mathrm{D}_{2} \mathrm{O}$ & $58 \pm 4$ & $1783 \pm 118$ & $2.8 \pm 0.2$ & $6 / 16 / 8146$ \\
\hline mMaple live & $50 \mathrm{mM}$ Tris in $\mathrm{D}_{2} \mathrm{O}$ & $56 \pm 3$ & $1621 \pm 159$ & $2.9 \pm 0.1$ & $3 / 6 / 1343$ \\
\hline
\end{tabular}


Table 2

Buffers used in this work.

\begin{tabular}{|c|c|c|}
\hline Buffer & Composition & Reference \\
\hline $\begin{array}{l}\text { FB } \\
\text { Fixation buffer }\end{array}$ & $2.4 \%(\mathrm{w} / \mathrm{v})$ formaldehyde in PBS & \\
\hline $\begin{array}{l}\text { PB } \\
\text { Permeabilization buffer }\end{array}$ & $0.4 \%(\mathrm{v} / \mathrm{v})$ Triton $\mathrm{X}-100$ in PBS & \\
\hline $\begin{array}{l}\text { QS } \\
\text { Quenching solution }\end{array}$ & $100 \mathrm{mM} \mathrm{NH} 4 \mathrm{Cl}$ in PBS & \\
\hline $\begin{array}{l}\text { TRB } \\
\text { Transport buffer }\end{array}$ & $\begin{array}{l}20 \mathrm{mM} \text { HEPES pH } 7.5 \\
110 \mathrm{mM} \text { KAc } \\
1 \mathrm{mM} \text { EGTA } \\
250 \mathrm{mM} \text { Sucrose } \\
\text { in } \mathrm{H}_{2} \mathrm{O}\end{array}$ & $\begin{array}{l}\text { Pleiner et al., } 2015^{53} \\
\text { Göttfert et al., } 2017^{54}\end{array}$ \\
\hline $\begin{array}{l}\text { TBA } \\
\text { Transport buffer with BSA }\end{array}$ & $\begin{array}{l}1 \%(\mathrm{w} / \mathrm{v}) \mathrm{BSA} \\
\text { in TRB }\end{array}$ & $\begin{array}{l}\text { Pleiner et al., } 2015^{53} \\
\text { Göttfert et al., } 2017^{54}\end{array}$ \\
\hline
\end{tabular}




\section{Table 3}

List of yeast strains used in this study.

\begin{tabular}{|c|c|c|}
\hline Strain & Genotype & Source \\
\hline MKY0100 & MATa, his3 $\Delta 200$, leu2-3,112, ura3-52, lys2-801 & Kaksonen lab \\
\hline MKY0122 & MATa, his3 $\Delta 200$, leu2-3,112, ura3-52, lys2-801, ABP1-mEGFP::HIS3MX6 & Kaksonen lab \\
\hline Nup188-mMaple & MATa, his3 $\Delta 200$, leu2-3,112, ura3-52, lys2-801, NUP188-mMaple::HIS3MX6 & This study \\
\hline Nup82-mMaple & MATa, his3 $\Delta 200$, leu2-3,112, ura3-52, lys2-801, NUP82-mMaple::HIS3MX6 & This study \\
\hline Nup192-mMaple & MATa, his3 $\Delta 200$, leu2-3,112, ura3-52, lys2-801, NUP192-mMaple::HIS3MX6 & This study \\
\hline mMaple-Nic96 & MATa, his3 $\Delta 200$, leu2-3,112::GalL-ISce-natNT2, ura3-52, lys2-801, mMaple-NIC96 & This study \\
\hline Nic96-mMaple & MATa, his3 $\Delta 200$, leu2-3,112, ura3-52, lys2-801, NIC96-mMaple::HIS3MX6 & This study \\
\hline $\begin{array}{l}\text { Abp1-mEGFP } \\
\text { Nup188-mMaple }\end{array}$ & MATa, his3 $\Delta 200$, leu2-3,112, ura3-52, lys2-801, ABP1-mEGFP::HIS3MX6, NUP188-mMaple::hphNT1 & This study \\
\hline $\begin{array}{l}\text { mMaple-Nic96 } \\
\text { Nup49-mEGFP }\end{array}$ & $\begin{array}{l}\text { MATa, his3 } 200 \text {, leu2-3,112::GalL-ISce-natNT2, ura3-52, lys2-801, mMaple-NIC96, NUP49- } \\
\text { mEGFP::HIS3MX6 }\end{array}$ & This study \\
\hline $\begin{array}{l}\text { Nup188-mMaple } \\
\text { Nup82-mMaple }\end{array}$ & MATa, his3 $\Delta 200$, leu2-3,112, ura3-52, lys2-801, NUP188-mMaple::HIS3MX6, NUP82-mMaple::LEU2 & This study \\
\hline
\end{tabular}


Table 4

Acquisition parameters during SMLM imaging.

\begin{tabular}{llll}
\hline Sample & No. of frames & Frametime [ms] & Laser intensity [kW/cm $\left.{ }^{2}\right]$ \\
\hline U2OS Nup96-mEGFP Nanobodies & $\sim 60-90 \mathrm{k}$ & $15 / 20 / 30$ & $\sim 6$ \\
\hline U2OS Nup96-mEGFP Antibodies & $\sim 70-120 \mathrm{k}$ & 15 & $\sim 9$ \\
\hline U2OS Nup96-Halo O2-AF647 & $\sim 50 \mathrm{k}$ & 50 & $\sim 6$ \\
\hline U2OS Nup96-Halo O4-AF647 & $\sim 20-30 \mathrm{k}$ & 50 & $\sim 6$ \\
\hline U2OS Nup96-Halo Cy5 & $\sim 40-70 \mathrm{k}$ & 40 & $\sim 6$ \\
\hline U2OS Nup96-Halo PA-JF549 & $\sim 10 \mathrm{k}$ & 50 & $\sim 3.5$ \\
\hline U2OS Nup96-SNAP AF647 & $\sim 50-70 \mathrm{k}$ & $30 / 40$ & $\sim 6$ \\
\hline U2OS Nup96-mMaple & $\sim 10-50 \mathrm{k}$ & 50 & $\sim 3.5$ \\
\hline HEK Nup107-mMaple & $\sim 20-50 \mathrm{k}$ & 50 & $\sim 3.5$ \\
\hline Yeast NPC-mMaple & $\sim 100 \mathrm{k}$ & 25 & $\sim 3.5$ \\
\hline
\end{tabular}




\section{Table 5}

\section{Used imaging buffers.}

*GLOX/MEA with $100 \mathrm{mM}$ MEA instead of $35 \mathrm{mM}$ to decrease the fraction of fluorophores in their on-state to suitable level.

\begin{tabular}{|c|c|c|c|}
\hline Buffer & Composition & Samples & Reference \\
\hline $50 \mathrm{mM}$ Tris in $\mathrm{D}_{2} \mathrm{O}$ & $50 \mathrm{mM}$ Tris/ $\mathrm{HCl} \mathrm{pH} 8$ in $95 \%(\mathrm{v} / \mathrm{v}) \mathrm{D}_{2} \mathrm{O}$ & $\begin{array}{l}\text { U2OS Nup96-Halo PA-JF549 } \\
\text { U2OS Nup96-mMaple } \\
\text { HEK Nup107-mMaple } \\
\text { Yeast NPC-mMaple }\end{array}$ & Ong et al., $2015^{37}$ \\
\hline $50 \mathrm{mM}$ Tris in $\mathrm{H}_{2} \mathrm{O}$ & $50 \mathrm{mM}$ Tris/ $\mathrm{HCl} \mathrm{pH} 8$ in $\mathrm{H}_{2} \mathrm{O}$ & U2OS Nup96-mMaple & \\
\hline GLOX/MEA & $\begin{array}{l}50 \mathrm{mM} \text { Tris/HCl pH } 8 \\
10 \mathrm{mM} \mathrm{NaCl} \\
10 \%(\mathrm{w} / \mathrm{v}) \text { D-Glucose } \\
500 \mu \mathrm{g} / \mathrm{mL} \text { Glucose oxidase } \\
40 \mu \mathrm{g} / \mathrm{mL} \text { Glucose catalase } \\
35 \mathrm{mM} \mathrm{MEA} \mathrm{in} \mathrm{H}_{2} \mathrm{O}\end{array}$ & $\begin{array}{l}\text { U2OS Nup96-mEGFP Nanobodies } \\
\text { U2OS Nup96-mEGFP Antibodies* } \\
\text { U2OS Nup96-Halo O2-AF647 } \\
\text { U2OS Nup96-Halo O4-AF647 } \\
\text { U2OS Nup96-Halo Cy5 } \\
\text { U2OS Nup96-SNAP AF647 }\end{array}$ & Heilemann et al., $2005^{59}$ \\
\hline GLOX/BME & $\begin{array}{l}50 \mathrm{mM} \text { Tris/HCl } \mathrm{pH} 8 \\
10 \mathrm{mM} \mathrm{NaCl} \\
10 \%(\mathrm{w} / \mathrm{v}) \text { D-Glucose } \\
500 \mu \mathrm{g} / \mathrm{mL} \text { Glucose oxidase } \\
40 \mu \mathrm{g} / \mathrm{mL} \text { Glucose catalase } \\
143 \mathrm{mM} \mathrm{BME} \text { in } \mathrm{H}_{2} \mathrm{O}\end{array}$ & U2OS Nup96-SNAP AF647 & Bates et al., $2005^{60}$ \\
\hline GLOX/MEA in $\mathrm{D}_{2} \mathrm{O}$ & $\begin{array}{l}50 \mathrm{mM} \text { Tris/HCl } \mathrm{pH} 8 \\
10 \mathrm{mM} \mathrm{NaCl} \\
10 \%(\mathrm{w} / \mathrm{v}) \text { D-Glucose } \\
500 \mu \mathrm{g} / \mathrm{mL} \text { Glucose oxidase } \\
40 \mu \mathrm{g} / \mathrm{mL} \text { Glucose catalase } \\
35 \mathrm{mM} \mathrm{MEA} \\
\text { in } 90 \%(\mathrm{v} / \mathrm{v}) \mathrm{D}_{2} \mathrm{O}\end{array}$ & U2OS Nup96-SNAP AF647 & Klehs et al., $2014^{36}$ \\
\hline Sulfite/MEA & $\begin{array}{l}50 \mathrm{mM} \text { Tris/ } \mathrm{HCl} \mathrm{pH} 8 \\
50 \mathrm{mM} \mathrm{Na} 2 \mathrm{SO}_{3} / \mathrm{NaOH} \mathrm{pH} 8 \\
35 \mathrm{mM} \mathrm{MEA} \\
\text { in } \mathrm{H}_{2} \mathrm{O}\end{array}$ & U2OS Nup96-SNAP AF647 & Hartwich et al., $2018^{35}$ \\
\hline
\end{tabular}

